\title{
THE FREE BOUNDARY OF A SEMILINEAR ELLIPTIC EQUATION ${ }^{1}$
}

BY

\author{
AVNER FRIEDMAN AND DANIEL PHILLIPS
}

\begin{abstract}
The Dirichlet problem $\Delta u=\lambda f(u)$ in a domain $\Omega, u=1$ on $\partial \Omega$ is considered with $f(t)=0$ if $t \leqslant 0, f(t)>0$ if $t>0, f(t) \sim t^{p}$ if $t \downarrow 0,0<p<1 ; f(t)$ is not monotone in general. The set $\{u=0\}$ and the "free boundary" $\partial\{u=0\}$ are studied. Sharp asymptotic estimates are established as $\lambda \rightarrow \infty$. For suitable $f$, under the assumption that $\Omega$ is a two-dimensional convex domain, it is shown that $\{u=0\}$ is a convex set. Analogous results are established also in the case where $\partial u / \partial \nu+$ $\mu(u-1)=0$ on $\partial \Omega$.
\end{abstract}

Introduction. In this paper we study the Dirichlet problem

$$
\begin{aligned}
\Delta u & =\lambda f(u) \quad \text { in } \Omega \quad(\lambda>0), \\
u & =1 \quad \text { on } \partial \Omega
\end{aligned}
$$

where $\Omega$ is a bounded domain in $R^{n}$ and

$$
\begin{aligned}
& f(t)=0 \quad \text { if } t \leqslant 0, \quad f(t)>0 \quad \text { if } t>0, \\
& f(t) \sim t^{p} \quad \text { as } t \downarrow 0, \quad 0<p<1 ;
\end{aligned}
$$

$f(t)$ is not assumed to be monotone. The motivation for this problem comes from reaction-diffusion models in which

$$
f(t) \uparrow \quad \text { if } 0<t<t_{0}, \quad f(t) \downarrow \quad \text { if } t_{0}<t<1
$$

for some $t_{0} \in(0,1)$; see $[2,3,7,18,19]$.

The solution of $(0.1)$ has, in general, more than one solution. We are interested primarily in those solutions which are either minimal, or maximal, or minimizers of the functional

$$
\int_{\Omega}\left(\frac{1}{2}|\nabla v|^{2}+\lambda F(v)\right) d x \quad\left(F(t)=\int_{0}^{t} f(s) d s\right)
$$

subject to the boundary condition $v=1$.

We shall study the nature of the "dead core" $N_{\lambda}=\left\{u_{\lambda}=0\right\}\left(u_{\lambda}\right.$ is a solution of $(0.1))$; if $p \geqslant 1$ then the dead core is empty, by the maximum principle. One of the

Received by the editors January 10, 1983.

1980 Mathematics Subject Classification. Primary 35J20, 35R35; Secondary 35B05.

Key words and phrases. Semilinear elliptic equation, free boundary, variational inequality, maximal and minimal solutions, minimizer, asymptotic behavior, reduced boundary, nonnegative mean curvature.

'This work is partially supported by National Science Foundation Grants MCS-8300293 and MCS8201036 
main results is that if $\Omega$ is a two-dimensional convex domain then $N_{\lambda}$ is a convex set provided $f$ satisfies

$$
f^{\prime}(t)+\frac{f(t)}{1-t}>0 \quad \text { for } 0<t \leqslant 1,
$$

i.e. every tangent line to $y=f(t)$ intersects the line $\{t=1\}$ in $\{y>0\}$ (this condition is satisfied for some reaction-diffusion models). Convexity of level surfaces $\left\{u_{\lambda}=c\right\}$ was proved by Caffarelli and Spruck [4] in case $f(t)$ is monotone increasing and negative for $0 \leqslant t<1$, and $f(1)=0$; our results and methods are unrelated to theirs; see Remark 5.1.

In $\S 1$ we show that for any two minimizers $u_{1}$ and $u_{2}$, either $u_{1} \geqslant u_{2}$ or $u_{2} \geqslant u_{1}$ and one of the sets $N_{u_{1}}, N_{u_{2}}$ is contained in the interior of the other. We also establish comparison theorems for minimal solutions, maximal solutions, and for minimizers corresponding to different $f$ 's and different $\Omega$ 's.

In $\S 2$ we establish uniqueness of solutions of $(0.1)$ under the assumption (0.3).

In $\$ 3$ we show that, for $\lambda$ large and any solution $u_{\lambda}$ of $(0.1), \partial N_{\lambda}$ is a smooth surface parallel to $\partial \Omega$ at distance $\gamma / \sqrt{\lambda}+O(1 / \lambda), \gamma$ constant.

In $\$ 4$ we specialize to domains $\Omega$ with $\partial \Omega$ having nonnegative mean curvature and establish a convexity type property of the boundary $\partial N_{\lambda}$. For a smooth portion of $\partial N_{\lambda}$ it asserts that the mean curvature is nonnegative.

In $\$ 5$ we establish, for two-dimensional convex domains, the convexity of the components of $N_{\lambda}$; under the assumption (0.3) $N_{\lambda}$ is shown to have at most one component.

Next, in $\$ 6$ we shall consider the limit case $p=0$, i.e., $f(t)=\chi_{[t>0]}$ and establish convexity of the coincidence set for some variational inequalities.

Finally, in $\$ 7$ we extend the results of the preceding sections to the case of the Robin problem, replacing the condition $u=1$ on $\partial \Omega$ by

$$
\frac{\partial u}{\partial \nu}+\mu(u-1)=0 \quad \text { on } \partial \Omega \quad(\mu>0)
$$

It is actually this case which primarily arises in applications [2,7]; the Dirichlet problem is viewed as a limit case when $\mu \rightarrow \infty$.

1. Comparison theorems. Let $f(t)$ be a function satisfying

$$
\begin{aligned}
& f(t)= \begin{cases}t^{p} f_{0}(t) & \text { if } 0 \leqslant t<\infty, \text { for some } 0<p<1, \\
0 & \text { if }-\infty<t<0,\end{cases} \\
& m \leqslant f_{0}(t) \leqslant M, \quad 0<m \leqslant M<\infty, \quad\left|f_{0}^{\prime \prime}(t)\right| \leqslant K
\end{aligned}
$$

where $m, M, K$ are positive constants; $f(t)$ is not assumed to be monotone increasing. We are interested in solutions of the Dirichlet problem

$$
\begin{aligned}
\Delta u & =f(u) \quad \text { in } \Omega, \\
u & =1 \quad \text { on } \partial \Omega
\end{aligned}
$$

where $\Omega$ is a bounded domain in $R^{n}$ with $C^{2+\alpha_{0}}$ boundary $\partial \Omega$. One can easily show that there exists a solution $u$ of $(1.2)$ and it belongs to $C^{2+\alpha}(\bar{\Omega})$, where $\alpha=$ $\min \left(p, \alpha_{0}\right)$. 
Since $\Delta u \geqslant 0$ in $\Omega$ and $u=1$ on $\partial \Omega$, the maximum principle gives

$$
u<1 \text { in } \Omega \text {. }
$$

We also have

$$
u \geqslant 0 \text { in } \Omega .
$$

Indeed, otherwise $u$ takes negative minimum in the nonempty open set $G=\{u<0\}$ $\cap \Omega$. Since, however, $\Delta u=f(u)=0$ in $G$, the maximum principle gives $u \equiv$ const in $G$, which is impossible.

Set $F(t)=\int_{0}^{t} f(s) d s$ and consider the functional

$$
J(v)=\int_{\Omega}\left(\frac{1}{2}|\nabla v|^{2}+F(v)\right) d x
$$

over the class of admissible functions

$$
K=\left\{v \in H^{1,2}(\Omega), v=1 \text { on } \partial \Omega\right\} .
$$

Problem $(\mathrm{J})$. Find $u \in K$ such that $J(u)=\min _{v \in K} J(v)$. If $u$ is a solution of this problem then $u$ is clearly also a solution of (1.2).

Definition 1.1. A solution of Problem $(\mathrm{J})$ is called a minimizer solution of (1.2).

We shall need some standard comparison lemmas.

LEMMA 1.1. Let $w \in C^{0}(\bar{\Omega})$,

$$
\begin{aligned}
\Delta w+b_{i} w_{x_{i}}+c w & =h & & \text { in } \Omega, \\
w & =\phi & & \text { on } \partial \Omega
\end{aligned}
$$

where $b_{i}$ are bounded functions, and suppose that $\Omega \subset\left\{\bar{x}<x_{1}<\bar{x}+a\right\}$ and $\sup _{\Omega} c(x)<\left(e^{\gamma a}-1\right)^{-1}$ where $\gamma>1, \gamma^{2}>\left(\sup _{\Omega}\left|b_{1}\right|\right) \gamma+1$. Then

$$
\begin{gathered}
w \geqslant \frac{\min _{\partial \Omega} \phi-\left(\sup _{\Omega} h\right)\left(e^{\gamma a}-1\right)}{1-\left(\sup _{\Omega} c\right)\left(e^{\gamma a}-1\right)} . \\
\max _{\bar{\Omega}}|w| \leqslant \frac{\max _{\partial \Omega}|\phi|+\left(\sup _{\Omega}|h|\right)\left(e^{\gamma a}-1\right)}{1-\left(\sup _{\Omega} c\right)\left(e^{\gamma a}-1\right)} .
\end{gathered}
$$

For the proof see [6, p. 330]. Notice that $c(x)$ is not required to be contınuous or bounded from below in $\Omega$.

COROllary 1.2. Suppose

$$
\begin{gathered}
\Delta u-h_{1}(u)|\nabla u|^{2} \leqslant h_{2}(u) \quad \text { in } \Omega, \\
\Delta v-h_{1}(v)|\nabla v|^{2} \geqslant h_{2}(v) \quad \text { in } \Omega, \\
u \geqslant v \quad \text { on } \partial \Omega
\end{gathered}
$$

where $h_{i}(t)$ are monotone increasing in $t$. Then $u \geqslant v$ in $\Omega$.

Indeed, the function $w=u-v$ satisfies

$$
\Delta w-h_{1}(u) \nabla(u+v) \cdot \nabla w-\left(c_{1}|\nabla v|^{2}+c_{2}\right) w \leqslant 0
$$


where

$$
c_{i}(x)= \begin{cases}\frac{h_{i}(u(x))-h_{i}(v(x))}{u(x)-v(x)} & \text { if } u(x) \neq v(x), \\ 0 & \text { if } u(x)=v(x)\end{cases}
$$

Since $c_{i} \geqslant 0$, we can apply (1.7).

LEMMA 1.3. If

$$
\begin{aligned}
\Delta v+c(x) v \leqslant 0 & \text { in } \Omega, \\
v \geqslant 0 & \text { in } \Omega, \quad v \neq 0,
\end{aligned}
$$

then $v>0$ in $\Omega$.

Indeed, writing $c=c^{+}-c^{-}$and noting that $c^{+} v \geqslant 0$, we get $\Delta v-c^{-}(x) v \leqslant 0$ in $\Omega$, so that $v>0$ by the strong maximum principle.

We now return to Problem (J).

THEOREM 1.4. If $u, v$ are two minimizers and $u \neq v$, then either $u>v$ in $\{v>0\}$ or $v>u$ in $\{u>0\}$.

Proof. Notice that $u \wedge v=\min \{u, v\}$ and $u \vee v=\max \{u, v\}$ belong to $K$ and therefore $J(u \wedge v) \geqslant J(u)=J(v), J(u \vee v) \geqslant J(u)$. Since, as easily seen, $J(u \wedge v)$ $+J(u \vee v)=J(u)+J(v)$, we conclude that $J(u \wedge v)=J(u)$. Consequently, the function $w=u \wedge v$ satisfies $\Delta w=f(w)$ in $\Omega$.

Next, $u-w \geqslant 0$ in $\Omega$ and $\Delta(u-w)=f(u)-f(w)=c(u-w)$, where $c=c(x)$ is a bounded function in any compact subset of $\{w>0\}$. By Lemma 1.3 we then have

$$
\begin{aligned}
& \text { either } u-w>0 \text { in }\{w>0\}, \text { i.e., } v<u \text { in }\{v>0\} \\
& \text { or } u-w \equiv 0 \text { in }\{w>0\}, \text { i.e., } u \leqslant v \text { in }\{u>0\}
\end{aligned}
$$

In the second case we repeat the preceding argument with $v, u$ (instead of $u, w)$ and deduce that $v>u$ in $\{u>0\}$.

THEOREM 1.5. If $w_{1}, w_{2}$ are two solutions of $(0.1)$ corresponding to $\lambda_{1}$ and $\lambda_{2}$ respectively, and if $\lambda_{1} \leqslant \lambda_{2}, w_{2} \leqslant w_{1}$ then either $w_{2} \equiv w_{1}$ and $\lambda_{2}=\lambda_{1}$ or $w_{2}<w_{1}$ on $\left\{w_{2}>0\right\}$.

Proof. Suppose there exists a point $x_{0} \in \Omega$ such that $0<w_{1}\left(x_{0}\right)=w_{2}\left(x_{0}\right)$. If $\lambda_{1}<\lambda_{2}$, then

$$
\Delta\left(w_{2}-w_{1}\right)\left(x_{0}\right)=\left(\lambda_{2}-\lambda_{1}\right) f\left(w_{2}\left(x_{0}\right)\right)>0
$$

which contradicts the fact that $w_{2}-w_{1}$ attains its maximum at $x_{0}$.

If $\lambda_{1}=\lambda_{2}$ then $\Delta\left(w_{2}-w_{1}\right)=\lambda_{2} c(x)\left(w_{2}-w_{1}\right)$, where

$$
c(x)= \begin{cases}\frac{f\left(w_{2}\right)-f\left(w_{1}\right)}{w_{2}-w_{1}} & \text { if } w_{2}-w_{1} \neq 0, \\ 0 & \text { if } w_{2}-w_{1}=0 .\end{cases}
$$


By Lemma $1.3, w_{2}-w_{1} \equiv 0$ in a neighborhood of $x_{0}$. Hence the set $\left\{x ; w_{2}=w_{1}\right\}$ is open and, consequently, $w_{2} \equiv w_{1}$.

Definition 1.2. The set $N_{u}=\{u=0\} \cap \Omega$ is called the dead core of $u$; this terminology is taken from the reaction-diffusion problem which is modelled by (1.2).

Notice that (1.1) implies that

(1.9) $f(t)$ is strictly increasing in some interval $0<t<\varepsilon_{0}$.

THEOREM 1.6. If $u, v$ are two solutions of $(0.1)$ with $u \leqslant v, u \neq v$, and if $N_{v}$ is nonempty, then $N_{v} \subset$ int $N_{u}$, i.e.,

$$
\operatorname{dist}\left(N_{v}, R^{n} \backslash N_{u}\right)>0 .
$$

Proof. Set $G_{t}=\{u<t\}$ for some small $t>0$. By Theorem 1.5, $v \geqslant u+\delta$ on $\partial G_{t}$, for some $\delta>0$. Suppose (1.10) is not true. Then for any $\varepsilon>0$ there is a unit vector $e$ such that the function $v_{\varepsilon}(x)=v(x+\varepsilon e)$ satisfies

$$
v_{\varepsilon}=0<u \text { at some point } x_{\varepsilon} \in G_{t} .
$$

Now, if $\varepsilon$ is small enough (depending on $\delta$ ) then

$$
v_{\varepsilon} \geqslant u+\delta / 2>u \quad \text { on } \partial G_{t} \text {. }
$$

Also,

$$
\Delta u=f(u), \quad \Delta v_{\varepsilon}=f\left(v_{\varepsilon}\right) \text { in } G_{t},
$$

and $f(s)$ is monotone increasing in $s$ in the range of $u(x)$ and of $v_{\varepsilon}(x), x \in G_{t}$, provided $t$ is sufficiently small at the outset. Thus Corollary 1.2 (with $h_{1} \equiv 0$ ) can be applied to (1.12), (1.13), and we conclude that $v_{\varepsilon} \geqslant u$ in $G_{t}$, which contradicts (1.11).

We shall denote the functional in (1.5) also by $J_{f}(v)$ in order to emphasize the dependence on $f$; the corresponding Problem $(\mathrm{J})$ is denoted by $\left(J_{f}\right)$.

THEOREM 1.7. Suppose $\tilde{f}$ satisfies the same properties as $f$ in (1.1) and let $u, \tilde{u}$ be minimizers of $\left(J_{f}\right)$ and $\left(J_{\tilde{f}}\right)$ respectively. If $\tilde{f}(t)>f(t)$ for all $0<t<1$ then $\tilde{u}<u$ in $\{\tilde{u}>0\}$ and $\operatorname{dist}\left(N_{u}, R^{n} \backslash N_{\tilde{u}}\right)>0$.

Proof. Denote $J_{f}(v)$ by $J(v)$ and $J_{\tilde{f}}(v)$ by $\tilde{J}(v)$. Let $\tilde{F}(t)=\int_{0}^{t} \tilde{f}(s) d s$. Then we have $F(t)-F(s)<\tilde{F}(t)-\tilde{F}(s)$ if $0 \leqslant s<t<1$. Consequently,

$$
\tilde{F}(u \wedge \tilde{u})+F(u \vee \tilde{u})<\tilde{F}(\tilde{u})+F(u)
$$

at each point $x$ for which $\tilde{u}(x)>u(x)$. If $\tilde{u}(x) \leqslant u(x)$ then equality holds in (1.14). Thus, provided the set $\{\tilde{u}>u\}$ is nonempty we have

$$
\int_{\Omega} \tilde{F}(u \wedge \tilde{u})+\int_{\Omega} F(u \vee \tilde{u})<\int_{\Omega} \tilde{F}(\tilde{u})+\int_{\Omega} F(u)
$$

and, consequently, also

$$
\tilde{J}(u \wedge \tilde{u})+J(u \vee \tilde{u})<J(\tilde{u})+J(u) .
$$

Since, however, $u \wedge \tilde{u}$ and $u \vee \tilde{u}$ belong to $K$, we must have $\tilde{J}(u \wedge \tilde{u}) \geqslant \tilde{J}(\tilde{u})$ and $J(u \vee \tilde{u}) \geqslant J(u)$, contradicting (1.15).

We have thus proved that $u \geqslant \tilde{u}$ in $\Omega$. We can now proceed as in Theorems 1.4 and 1.5 in order to deduce that $u>\tilde{u}$ in $\{\tilde{u}>0\}$ and that $N_{u} \subset$ int $N_{\tilde{u}}$. 
DEFINITION 1.3. A solution $\bar{u}_{\lambda}\left(\underline{u}_{\lambda}\right)$ of $(0.1)$ is called maximal (minimal) if for any solution $u_{\lambda}$ of $(0.1)$ there holds $\bar{u}_{\lambda} \geqslant u_{\lambda}$ (respectively, $\underline{u}_{\lambda} \leqslant u_{\lambda}$ ).

THEOREM 1.8. For each $\lambda>0$ there exist maximal and minimal solutions of $(0.1)$.

Proof. We shall apply a monotone iteration method as in Keller [10]. Choose $M>0$ such that the function $h(u)=\lambda f(u)-M u^{p}$ satisfies

$$
\begin{array}{ll}
h(u) \leqslant 0 & \text { if } 0 \leqslant u \leqslant 1, \\
h^{\prime}(u) \leqslant 0 & \text { if } 0<u \leqslant 1 .
\end{array}
$$

Introduce the functional

$$
H_{q}(v)=\int_{\Omega}\left[\frac{1}{2}|\nabla v|^{2}+\frac{M}{p+1}|v|^{p+1}+h(q(w)) v\right] d v
$$

for a given function $q \in C^{1}(\bar{\Omega}), 0 \leqslant q(x) \leqslant 1$, and consider the problem: Find $z$ satisfying

$$
\min _{v \in K} H_{q}(v)=H_{q}(z), \quad z \in K .
$$

Since the functional is lower semicontinuous, there exists a solution $z=z_{q}$ of (1.17), and since the function

$$
A(p, z, x)=\frac{|p|^{2}}{2}+\frac{M}{p+1}|z|^{p+1}+h(q(x)) z
$$

is convex in $(p, z)$, the solution is unique.

The inequality $h \leqslant 0$ implies that $H_{q}(\max (z, 0)) \leqslant H_{q}(z)$; hence the minimizer $z$ is nonnegative. Finally, since $h$ is nonincreasing, if $z>1$ then

$$
\begin{aligned}
A_{z}(p, z, x) & =M z^{p}+h(q(x)) \geqslant M z^{p}+h(1) \\
& =M z^{p}-M+\lambda f(1)>0 .
\end{aligned}
$$

Therefore $H_{q}(\min (z, 1)) \leqslant H_{q}(z)$, so that $z \leqslant 1$. We have thus proved that the minimizer of (1.17) satisfies

$$
0 \leqslant z \leqslant 1
$$

Clearly also

$$
\begin{aligned}
\Delta z-M z^{p} & =h(q(x)) \quad \text { in } \Omega, \\
z & =1 \quad \text { on } \partial \Omega
\end{aligned}
$$

and $z \in C^{2+\alpha}(\bar{\Omega})$.

We now take $w_{0} \equiv 1$ and define inductively $w_{m}$ as the solution of (1.17) with $q=w_{m-1}$. By (1.18),

$$
w_{1} \leqslant w_{0} .
$$

Assuming inductively that $w_{i} \leqslant w_{i-1}$ for $i \leqslant m-1$, we proceed to show that

$$
w_{m} \leqslant w_{m-1} \text {. }
$$

First

$$
\begin{gathered}
\Delta\left(w_{m}-w_{m-1}\right)=M\left(w_{m}^{p}-w_{m-1}^{p}\right)+h\left(w_{m-1}\right)-h\left(w_{m-2}\right) \text { in } \Omega, \\
w_{m}-w_{m-1}=0 \text { on } \partial \Omega .
\end{gathered}
$$


Since $h$ is nonincreasing we get

$$
\Delta\left(w_{m}-w_{m-1}\right) \geqslant M\left(w_{m}^{p}-w_{m-1}^{p}\right) \text { in } \Omega
$$

hence $w_{m}-w_{m-1}$ cannot take a negative minimum in $\Omega$, and (1.21) follows.

From (1.20), (1.21) and (1.18) we get

$$
0 \leqslant w_{m} \leqslant w_{m-1} \leqslant 1 .
$$

By elliptic estimates, $\left|w_{m}\right|_{C^{2 . \alpha}(\bar{\Omega})} \leqslant C$. Hence $w_{m} \rightarrow u$ in $C^{2}(\bar{\Omega}), u \in C^{2+\alpha}(\bar{\Omega})$, and $\Delta u=M u^{p}+h(u)=M u^{p}+\lambda f(u)-M u^{p}$ in $\Omega$, i.e., $u$ is a solution of (0.1).

We claim that $u$ is a maximal solution. Indeed, if $v$ is any solution of $(0.1)$ then $v \leqslant 1=w_{0}$. Writing

$$
\Delta\left(w_{m+1}-v\right)=M\left(w_{m+1}^{p}-v^{p}\right)+h\left(w_{m}\right)-h(v)
$$

we easily deduce (cf. the proof of (1.21)) that if $w_{m} \geqslant v$ in $\Omega$ then $w_{m+1} \geqslant v$ in $\Omega$. It follows that $u=\lim w_{m} \geqslant v$.

Similarly, starting with $z_{0}=0$ solve (1.17) successively with $q=z_{m-1}, z=z_{m}$ and obtain a sequence $0 \leqslant z_{m-1} \leqslant z_{m} \leqslant 1$ with $z=\lim z_{m}$ a minimal solution.

Added in proof. It has been brought to our attention that Theorem 1.8 is a special case of a result of H. Amann (Indiana Univ. Math. J. 21 (1971), 123-146).

THEOREM 1.9. If $\lambda_{1}<\lambda_{2}$ then

$$
\begin{array}{lll}
\bar{u}_{\lambda_{2}}<\bar{u}_{\lambda_{1}} & \text { on }\left\{\bar{u}_{\lambda_{2}}>0\right\}, & N_{\bar{u}_{\lambda_{1}}} \subset \text { int } N_{\bar{u}_{\lambda_{2}}}, \\
\underline{u}_{\lambda_{2}}<\underline{u}_{\lambda_{1}} \text { on }\left\{\underline{u}_{\lambda_{2}}>0\right\}, & N_{\underline{u}_{\lambda_{1}}} \subset \text { int } N_{\underline{u}_{\lambda_{2}}} .
\end{array}
$$

Proof. Let $w_{m}$ be the sequence constructed in the preceding proof for $\lambda=\lambda_{1}$. Then $\bar{u}_{\lambda_{2}} \leqslant 1=w_{0}$. We can proceed inductively to show that $w_{m+1}-\bar{u}_{\lambda_{2}} \geqslant 0$. Indeed, if $w_{m}-\bar{u}_{\lambda_{2}} \geqslant 0$ then, since $\lambda_{1}-\lambda_{2}<0$,

$$
\begin{aligned}
\Delta\left(w_{m+1}-\bar{u}_{\lambda_{2}}\right) & =M\left(w_{m+1}^{p}-\bar{u}_{\lambda_{2}}^{p}\right)+h\left(w_{m}\right)-h\left(\bar{u}_{\lambda_{2}}\right)+\left(\lambda_{1}-\lambda_{2}\right) f\left(\bar{u}_{\lambda_{2}}\right) \\
& \leqslant M\left(w_{m+1}^{p}-\bar{u}_{\lambda_{2}}^{p}\right)
\end{aligned}
$$

and the maximum principle yields $w_{m+1}-\bar{u}_{\lambda_{2}} \geqslant 0$. It follows that $\bar{u}_{\lambda_{1}} \geqslant \bar{u}_{\lambda_{2}}$.

Similarly one shows that $\underline{u}_{\lambda_{1}} \geqslant \underline{u}_{\lambda_{2}}$, and the rest follows from Theorems 1.5 and 1.6 .

THEOREM 1.10. (i) If $\Omega$ is a ball $B_{R}(0)$ and $u$ a minimal or a maximal solution, then $u(x)=u(|x|)$;

(ii) if $u$ is a minimal or a maximal solution of

$$
\begin{aligned}
\Delta u & =\lambda f(u) \quad \text { in } \tilde{\Omega}=\left\{R_{1}<|x|<R_{2}\right\}, \\
u & =1 \quad \text { if }|x|=R_{1}, \\
u & =0 \quad \text { if }|x|=R_{2}
\end{aligned}
$$

where $0<R_{1}<R_{2}$, then $u(x)=u(|x|)$.

Notice that the existence of minimal and maximal solutions of (1.23) is established as in Theorem 1.8. 
Proof. If $T$ is an orthogonal transformation and $w_{m}$ is the sequence which converges to a maximal solution (as in Theorem 1.8) then by uniqueness $w_{m}(T x)=$ $w_{m}(x)$. Hence also $u(T x)=u(x)$ for the maximal solution, so that $u(x)=u(|x|)$.

THEOREM 1.11. Let $\bar{u}, \underline{u}$ be the maximal and minimal solutions of (0.1) and $\bar{v}, \underline{v}$ the maximal and minimal solutions for a ball $B_{R}\left(x^{0}\right)$ in $\Omega$ and a shell $S_{R_{1}, R_{2}}\left(y^{\overline{0}}\right)=$ $B_{R_{2}}\left(y^{0}\right) \backslash \widehat{B_{R_{1}}\left(y^{0}\right)}$ with $B_{R_{1}}\left(y^{0}\right) \subset R^{n} \backslash \Omega$ and zero boundary values on the outer surface. Then

$$
\begin{aligned}
& \bar{u} \leqslant \bar{v} \quad \text { in } B_{R}\left(x^{0}\right), \\
& \underline{v} \leqslant \underline{u} \quad \text { in } S_{R_{1}, R_{2}}\left(y^{0}\right) \cap \Omega .
\end{aligned}
$$

Proof. To prove (1.24), we take as usual the sequences $\bar{u}_{m}, \bar{v}_{m}$ which converge to $\bar{u}$ and $\bar{v}$, respectively, and assume inductively that $\bar{u}_{m} \leqslant \bar{v}_{m}$ on $B_{R}\left(x^{0}\right)$ (trivially $\left.\bar{u}_{0}=\bar{v}_{0}=1\right)$. Thus

$$
\begin{aligned}
\Delta\left(\bar{u}_{m+1}-\bar{v}_{m+1}\right) & =M\left(\bar{u}_{m+1}^{p}-\bar{v}_{m+1}^{p}\right)+h\left(\bar{u}_{m}\right)-h\left(\bar{v}_{m}\right) \\
& \geqslant M\left(\bar{u}_{m+1}^{p}-\bar{v}_{m+1}^{p}\right) \quad \text { in } B_{R}\left(x^{0}\right)
\end{aligned}
$$

and $\bar{u}_{m+1}-\bar{v}_{m+1} \leqslant 0$ on $\partial B_{R}\left(x^{0}\right)$. It follows that $\bar{u}_{m+1}-\bar{v}_{m+1} \leqslant 0$ in $B_{R}\left(x^{0}\right)$. Taking $m \rightarrow \infty$, (1.24) follows. The proof of (1.25) is similar.

REMARK. Theorem 1.11 can be extended to minimizers as well as to general comparison domains (instead of just balls and shells). However, only Theorem 1.11 will be needed later on.

\section{Uniqueness of solutions.}

THEOREM 2.1. If

$$
f^{\prime}(t)+\frac{f(t)}{1-t}>0 \quad(0<t \leqslant 1)
$$

then the solution of (1.2) is unique.

This theorem is due to Cohen and Laetsch [5] whose proof depends on the concept of maximal solutions. We give here another proof which can be extended to general Dirichlet boundary conditions.

Proof. Let $u$ be a solution of (1.2) and define a function $w$ by $u=g(w)$, where $g(t)=1-t^{\beta}(\beta>1)$. Then $w=(1-u)^{1 / \beta}$ for $0 \leqslant u \leqslant 1$. We compute

$$
\Delta w+\frac{g^{\prime \prime}(w)}{g^{\prime}(w)}|\nabla w|^{2}-\frac{f(g(w))}{g^{\prime}(w)}=0 \text {. }
$$

If

$$
\left(\frac{g^{\prime \prime}(t)}{g^{\prime}(t)}\right)^{\prime} \leqslant 0, \quad\left(\frac{f(g(t))}{g^{\prime}(t)}\right)^{\prime} \geqslant 0
$$

then, by Corollary 1.2, there exists at most one solution $w$ of (2.2) with $w=0$ on $\partial \Omega$; this gives the assertion of the theorem. To prove (2.3) we easily check that

$$
\frac{g^{\prime \prime}(t)}{g^{\prime}(t)}=\frac{\beta-1}{t}, \quad \text { so that }\left(\frac{g^{\prime \prime}}{g^{\prime}}\right)^{\prime} \leqslant 0 \text {. }
$$


The second inequality in $(2.3)$ reduces to $\left(f\left(1-t^{\beta}\right) / t^{\beta-1}\right)^{\prime} \leqslant 0$ or, with $s=1-t^{\beta}$, to

$$
f^{\prime}(s)+\frac{\beta-1}{\beta} \frac{f(s)}{1-s} \geqslant 0 .
$$

But this is a consequence of (2.1) if $\beta$ is large enough.

REMARK 2.1. If $f(t)$ is concave then $f(1)-f(u) \leqslant(1-u) f^{\prime}(u)$; consequently $f^{\prime}(u)+f(u) /(1-u)>f(1) /(1-u)$. Thus if $f(1)>0$ then $f$ satisfies $(2.1)$ and thus (1.2) has at most one solution. If $f(1)=0$ then the solution is not unique, in general. Indeed, if $n=1, \Omega=\{-a<x<a\}$ then (1.2) reduces to

$$
\begin{gathered}
u^{\prime \prime}=f(u) \quad \text { for } 0<x<a, \\
u^{\prime}(0)=0, \quad u(a)=1 .
\end{gathered}
$$

If $a$ is sufficiently large then the solution satisfies: $u(0)=0$ and $u^{\prime}=\sqrt{2 F(u)}$ and we can construct a concave $f(u)$ such that (1.1) holds with $m=0$ and $u(0)=0$, $u(a)=1$. Another solution is given by $u \equiv 1$.

REMARK 2.2. We can also construct two solutions of (2.4) in case $f(1)>0$, with $f(t)$ as in (1.1), monotone increasing for $0<t<\theta_{*}$ and monotone decreasing for $\theta_{*}<t<1$. Indeed, let $\frac{1}{2}<\theta<\theta_{1}<1$ and choose $f(t)$ concave for $0<t<\theta$, $f(\theta)=2 \varepsilon, \varepsilon \leqslant f(t) \leqslant 2 \varepsilon$ and $f^{\prime}(t) \leqslant 0$ for $\theta<t<\theta_{1}, f(t)=\varepsilon$ if $\theta_{1}<t<1$. A solution of (2.4) with $u(0)=0$ exists provided $a$ is suitably chosen; $a$ depends on $\varepsilon$, but $0<\overline{\bar{a}} \leqslant a \leqslant \bar{a}<\infty$ where $\bar{a}, \overline{\bar{a}}$ are independent of $\varepsilon$, if $\varepsilon$ is small enough. Another solution of (2.4) is given by

$$
u(x)=1-\frac{\varepsilon a^{2}}{2}+\frac{\varepsilon x^{2}}{2}
$$

provided $\varepsilon$ is sufficiently small so that $1-\varepsilon a^{2} / 2>\theta_{1}$.

REMARK 2.3. If $f(t)$ is strictly concave with $f(1)=0$, then one can still prove uniqueness for the minimizer. Indeed, if $u$ and $v$ are two minimizers, set $w(t)=t u+$ $(1-t) v$ and suppose $u \geqslant v$. We compute that

$$
\frac{d}{d t} J(w(t))=\int\{f(t u+(1-t) v)-[t f(u)+(1-t) f(v)]\}(u-v)>0,
$$

so that $J(u)>J(v)$, a contradiction.

REMARK 2.4. Theorem 2.1 can be applied to the case

$$
f(t)=t^{p} \exp \left\{-\frac{\gamma}{\beta+1-\beta t}\right\} \quad(\gamma>0, \beta>0)
$$

which arise in reaction-diffusion $[\mathbf{2}, \mathbf{3}, \mathbf{7}, \mathbf{1 7}]$. The condition $(2.1)$ is satisfied for some range of the parameters $\beta, \gamma$.

THEOREM 2.2. If $\lambda$ is sufficiently small then there exists a unique solution $u$ of $(0.1)$ and $u>0$ in $\Omega$.

Proof. By (1.1),

$$
f(t)=f_{1}(t)+f_{2}(t)
$$

$$
f_{1}(t) \text { monotone increasing, }\left|f_{2}^{\prime}(t)\right| \leqslant C \text {. }
$$


Suppose $u, v$ are two solutions of $(0.1)$. Then

$$
\Delta(u-v)-\lambda\left(f_{1}(u)-f_{1}(v)\right)=\lambda\left(f_{2}(u)-f_{2}(v)\right)
$$

and $f_{1}(u)-f_{1}(v)=-c w$ where $w=u-v$ and $c \leqslant 0$. Since also $\left|f_{2}(u)-f_{2}(v)\right| \leqslant$ $C|u-v|$, we see that

$$
\begin{gathered}
\Delta w+\lambda c w=H, \quad|H| \leqslant \lambda C|w| \quad \text { in } \Omega, \\
w=0 \quad \text { in } \partial \Omega .
\end{gathered}
$$

Applying Lemma 1.1. we get $|w|_{L^{x}(\Omega)} \leqslant C \lambda|w|_{L^{x}(\Omega)}$; hence, if $C \lambda<1$ then $w \equiv 0$, which proves uniqueness.

By Lemma 1.1 we also have $u \geqslant 1-C \lambda \sup _{\Omega} f(u)>0$ if $\lambda$ is small enough, since $f(u) \leqslant$ const if $0 \leqslant u \leqslant 1$.

3. Asymptotic behavior as $\lambda \rightarrow \infty$. Let $u=u_{\lambda}$ be any solution of (0.1) and set $N_{\lambda}=N_{u_{\lambda}}$. We shall study the behavior of $N_{\lambda}$ as $\lambda \rightarrow \infty$.

Set

$$
\begin{gathered}
\Omega_{\delta}=\{x \in \Omega, \operatorname{dist}(x, \partial \Omega)>\delta\}, \\
B_{R}\left(x^{0}\right)=\left\{\left|x-x^{0}\right|<R\right\}, \quad B_{R}=B_{R}(0) .
\end{gathered}
$$

THEOREM 3.1. There exist positive constants $\gamma, c_{1}, c_{2}$ such that for any solution $u_{\lambda}$

$$
\Omega_{\gamma / \sqrt{\lambda}+c_{1} / \lambda} \subset N_{\lambda} \subset \Omega_{\gamma / \sqrt{\lambda}-c_{2} / \lambda}
$$

provided $\lambda$ is large enough.

PRoof. It is easy to establish the crude estimates

$$
\Omega_{C / \sqrt{\lambda}} \subset N_{\lambda} \subset \Omega_{c / \sqrt{\lambda}}
$$

where $C, c$ are positive constants independent of $\lambda$. Indeed, to prove the second part it suffices to show that $\left|D u_{\lambda}\right| \leqslant C \sqrt{\lambda}$. Since the function $w_{\lambda}(x)=u_{\lambda}(x / \sqrt{\lambda})$ satisfies $\Delta w_{\lambda}=f\left(w_{\lambda}\right)$ in $\Omega_{\lambda}\{x / \sqrt{\lambda} ; x \in \Omega\}$ and $0 \leqslant f \leqslant 1$, by elliptic estimates $\left|D w_{\lambda}\right| \leqslant C$ in $\Omega_{\lambda}$, so that, indeed, $\left|D u_{\lambda}\right| \leqslant C \sqrt{\lambda}$.

To prove the first inclusion in (3.2) we proceed as in [7] and consider the function

$$
v(x)=A\left|x-x_{0}\right|^{2 /(1-p)} \quad(A>0)
$$

where $x_{0} \in \Omega$, and set $d=\operatorname{dist}\left(x_{0}, \partial \Omega\right)$. Then $\Delta v \leqslant a_{0} A^{1-p_{v}}{ }^{p}$ in $\Omega$ and $v \geqslant 1$ on $\partial \Omega$ if $\operatorname{Ad}^{2 /(1-p)}=1$, where $a_{0}$ is a positive constant independent of $A$. Since $\Delta u_{\lambda} \geqslant$ $\lambda c\left(u_{\lambda}\right)^{p}(c>0)$, if $a_{0} A^{1-p}=\lambda c$ then, by comparison, $u_{\lambda} \leqslant v$; thus

$$
u_{\lambda}\left(x_{0}\right) \leqslant v\left(x_{0}\right)=0 \quad \text { if } d=\left(\frac{a_{0}}{c \lambda}\right)^{1 / 2},
$$

and the assertion follows.

The system

$$
\eta^{\prime}(s)=\sqrt{2 F(\eta)} \quad \text { for } s<0, \quad \eta(0)=1
$$


has a unique solution as long as $\eta(s)>0$; it determines a unique positive number $\gamma$ such that $\eta(-\gamma)=0$. Letting $\zeta(s)=\eta(\gamma+s)$ we have

$$
\begin{aligned}
\zeta^{\prime}(s) & =\sqrt{2 F(\zeta)} \text { for } 0<s<\gamma, \\
\zeta(0) & =0, \quad \zeta(s)>0 \quad \text { for } 0<s<\gamma, \\
\zeta(\gamma) & =1 .
\end{aligned}
$$

Let $\gamma \in \partial \Omega$ and let $B_{R}$ be a ball in $\Omega$ with $y \in \partial B_{R}$. Let $U$ be a maximal solution for $B_{R}$. By Theorems 1.10, 1.11, $U=U(r), u_{\lambda} \leqslant U$ : Furthermore, since $U$ is subharmonic and differentiable at $r=0, U^{\prime}(r) \geqslant 0$.

The function $U$ satisfies: $U^{\prime \prime}+(n-1) U^{\prime} / r=\lambda f(U)$ and the function

$$
Z(s)=U\left(R-\frac{\gamma_{0}}{\sqrt{\lambda}}+\frac{s}{\sqrt{\lambda}}\right) \quad\left(\gamma_{0} \text { to be determined }\right)
$$

satisfies

$$
Z^{\prime \prime}+\frac{n-1}{\rho \sqrt{\lambda}+s} Z^{\prime}=f(Z) \quad\left(\rho=R-\frac{\gamma_{0}}{\sqrt{\lambda}}\right) .
$$

Since $U^{\prime}(r) \geqslant 0$, the support of $Z(s)$ consists of one interval, namely $0 \leqslant s \leqslant \gamma_{0}$. From (3.2) (applied to $U$ in $B_{R}$ ) we have

$$
\gamma_{0} \leqslant c, \quad c \text { independent of } \lambda \text {. }
$$

Multiplying both sides of (3.4) by $Z^{\prime}(s)$, we get

$$
\frac{1}{2}\left(Z^{\prime 2}\right)^{\prime}+\frac{(n-1) Z^{\prime 2}}{\rho \sqrt{\lambda}+s}=(F(Z))^{\prime}
$$

Hence

$$
\left(Z^{\prime 2}\right)+\frac{C}{\sqrt{\lambda}} Z^{\prime 2} \geqslant 2(F(Z))^{\prime}, \quad C>0,
$$

where $C$ is independent of $\lambda$, by (3.5). From this we obtain

$$
\left(Z^{\prime 2} e^{C s / \sqrt{\lambda}}\right)^{\prime} \geqslant 2 e^{C s / \sqrt{\lambda}}(F(Z))^{\prime} .
$$

Integrating and using the relations $Z^{\prime}(0)=0, F(Z(0))=0$, we get

$$
\begin{aligned}
Z^{\prime 2}(s) & \geqslant 2 e^{-C s / \sqrt{\lambda}} \int_{0}^{s} e^{c t / \sqrt{\lambda}}\left(F(Z(t))^{\prime} d t\right. \\
& =2 F(Z)(s))-\frac{C}{\sqrt{\lambda}} \int_{0}^{s} e^{-c(s-t) / \sqrt{\lambda}} F(Z(t)) d t .
\end{aligned}
$$

Recalling that $Z^{\prime}(t) \geqslant 0$ we get $Z^{\prime}(s) \geqslant(1-C / \sqrt{\lambda})^{1 / 2} \sqrt{2 F(Z(s))}$. On the other hand, the function

$$
\tilde{\zeta}(s)=\zeta\left(s\left(1-\frac{C}{\sqrt{\lambda}}\right)^{1 / 2}\right)
$$


satisfies $\tilde{\zeta}^{\prime}(s)=(1-C / \sqrt{\lambda})^{1 / 2} \sqrt{2 F(\tilde{\zeta}(s))}$. By comparison we then deduce that

$$
Z(s) \geqslant \zeta\left(s\left(1-\frac{C}{\sqrt{\lambda}}\right)^{1 / 2}\right)
$$

Since $U(R)=1$ means $Z\left(\gamma_{0}\right)=1$, we conclude that $\gamma_{0}(1-C / \sqrt{\lambda})^{1 / 2} \leqslant \gamma$. Recalling that $u_{\lambda} \leqslant U$ we deduce that

$$
N_{\lambda} \supset \Omega_{R-\gamma_{0} / \sqrt{\lambda}} \supset B_{R-\gamma / \sqrt{\lambda}-c / \lambda} .
$$

Thus the first part of (3.1) follows.

To prove the second part of (3.1), we introduce the following shell $\tilde{\Omega}$ : The inner boundary is a sphere $S_{0}$ in $R^{n} \backslash \tilde{\Omega}$, with radius $R$, which contains a point $y$ on $\partial \Omega$, and the outer boundary is a sphere $S_{1}$ containing $\tilde{\Omega}$.

Let $V$ be the minimal solution for the shell $\tilde{\Omega}$ (cf. (1.23)). By Theorems 1.10, 1.11, $V=V(r), V \leqslant u_{\lambda}$; further, $V^{\prime}(r) \leqslant 0$ since $V$ is subharmonic. The function $\bar{Z}(s)=$ $V(R+\bar{\gamma} / \sqrt{\lambda}-s / \sqrt{\lambda})$ can be analyzed similarly to $Z(s)$. Thus we find that

$$
\bar{Z}(s) \leqslant \zeta\left(s\left(1+\frac{C}{\sqrt{\lambda}}\right)^{1 / 2}\right), \quad \bar{\gamma}\left(1+\frac{C}{\sqrt{\lambda}}\right)^{1 / 2} \geqslant \gamma,
$$

and this yields the second part of (3.1), if we let $y$ vary over $\partial \Omega$ in the above construction.

Definition 3.1. The set $\Gamma_{\lambda}=\partial\left\{u_{\lambda}>0\right\} \cap \Omega$ is called the free boundary of $u_{\lambda}$.

LEMMA 3.2. If $v$ is any solution of (1.2) then

$$
|\nabla v|^{2} \leqslant 2 F(v)+a v \text { in } \Omega
$$

where $a=2(n-1) \max _{x \in \partial \Omega}[|\nabla v(x)| \cdot K(x)]^{-}$and $K(x)$ is the mean curvature of $\partial \Omega$ at $x(K \geqslant 0$ if $\Omega$ is convex $)$.

For a proof see $[12,13,14,17,18]$.

THEOREM 3.3 (a) Suppose $u$ is a solution of $\Delta u=f(u)$ in $B_{1}(0)$ with $f$ satisfying $(1.1)$ and $0 \in \partial\{u>0\}$. There exist positive constants, $\alpha, \beta \geqslant 1, \sigma_{0}, \tau_{0}$ and $C$ depending on $n$ and the function $f$ such that $\left\{x ; x_{n}>\sigma \rho\right\} \cap B_{\rho}(0) \subset\{u=0\}$ with $\sigma \leqslant \sigma_{0}$ and $\rho \leqslant \tau_{0} \sigma^{\beta}$ implies that $B_{\rho / 4}(0) \cap \partial\{u>0\}$ is a graph of a $C^{1+\alpha}$ function $g$ in the direction $e_{n}$; moreover, with $x^{\prime}=\left(x_{1}, \ldots, x_{n-1}\right)$ there holds

$$
\begin{gathered}
\left|\nabla g\left(x^{\prime}\right)\right| \leqslant C \sigma \text { for }\left|x^{\prime}\right| \leqslant \frac{\rho}{8}, \\
\left|\nabla g\left(x_{1}^{\prime}\right)-\nabla g\left(x_{2}^{\prime}\right)\right| \leqslant C \sigma\left|\frac{x_{1}^{\prime}-x_{2}^{\prime}}{\rho}\right|^{\alpha} \text { for }\left|x_{1}^{\prime}\right|,\left|x_{2}^{\prime}\right| \leqslant \frac{\rho}{8} .
\end{gathered}
$$

(b) If $n=2$ and

$$
\limsup _{r \rightarrow 0} \frac{\left|\{u=0\} \cap B_{r}(0)\right|}{\left|B_{r}(0)\right|}>0
$$

where $|E|=n$-dimensional measure of $E$, then there exists a $\rho>0$ such that $B_{\rho}(0) \cap$ $\partial\{u>0\}$ is a $C^{1+\alpha}$ graph. 
For a proof see [1].

In the next theorem we improve Theorem 3.1. Let $x=h(t)\left(t=\left(t_{1}, \ldots, t_{n-1}\right)\right)$ be a $C^{1+\alpha}$ local parametrization of $\partial \Omega$ in a neighborhood of a point $x^{0}=h(0)$, and denote by $\nu(t)$ the inner normal to $\partial \Omega$ at $h(t)$.

THEOREM 3.4. There exist positive constants $\sigma_{1}, \sigma_{2}$ such that for any $\lambda$ sufficiently large and for any solution $u_{\lambda}$ of $(0.1), \Gamma_{\lambda}$ is a $C^{1+\delta_{1}}$ surface; furthermore, in terms of local coordinates $x=h(t), \Gamma_{\lambda} \cap B_{R}(h(0))$ can be represented (for small enough $R$ ) in the form $x=h(t)+k(t, \lambda) \nu(t)$ with $k(t, \lambda)$ satisfying, as a function of $t$,

$$
\begin{gathered}
\left|D_{t} k\right| \leqslant C / \lambda^{\delta_{1}} \quad\left(t \in \bar{B}_{R}(0)\right), \\
|k|_{C^{1+\delta_{2}\left(\bar{B}_{R}(0)\right)}} \leqslant C .
\end{gathered}
$$

Here $\bar{B}_{R}(0)$ is a ball of radius $R$ in the $(n-1)$-dimensional space. Notice that, by Theorem 3.1, $|k(t, \lambda)-\gamma / \sqrt{\lambda}| \leqslant C / \lambda$.

Proof. Consider first the case where

$$
h(t)=(t, \bar{h}(t)), \quad \bar{h}(0)=0, \quad \nabla \bar{h}(0)=0 .
$$

We introduce the mapping

$$
(t, s) \rightarrow x=\left(x^{\prime}, x_{n}\right)=\Phi(t, s) \equiv h(t)+s \nu(t) .
$$

Since $\partial \Omega$ is in $C^{2+\alpha_{0}}$, this is a $C^{1+\alpha_{0}}$ diffeomorphism from $\{(t, s) ;|t|<4 R, 0 \leqslant s \leqslant \bar{c}\}$ onto some $\bar{\Omega}$-neighborhood $V$ of

$$
\partial \Omega \cap\left\{x=\left(x^{\prime}, x_{n}\right) ; x^{\prime} \in \bar{B}_{3 R}(0), x_{n} \in(-c, c)\right\}
$$

for suitable $\bar{c}, c$.

From Theorem 3.2 it follows that

$$
\Gamma_{\lambda} \cap U \subset\left\{\Phi(t, s) ;|t|<4 R,\left|s-\frac{\gamma}{\sqrt{\lambda}}\right| \leqslant \frac{C}{\lambda}\right\} .
$$

Since $\bar{h}(0)=0$ and $\nabla \bar{h}(0)=0, x=\left(x^{\prime}, x_{n}\right)=(t, s)+O\left((|t|+|s|)^{1+\alpha_{0}}\right)$, i.e.,

$$
x^{\prime}=t+O\left((|t|+|s|)^{1+\alpha_{0}}\right), \quad x_{n}=s+O\left((|t|+|s|)^{1+\alpha_{0}}\right) .
$$

Consequently, $\Gamma_{\lambda} \cap\left[\bar{B}_{\gamma / 2 \sqrt{\lambda}}(0) \times(0 \leqslant s \leqslant 2 \gamma / \sqrt{\lambda})\right]$ is contained in

$$
\left\{x ;\left|x^{\prime}\right|<\frac{\gamma}{\sqrt{\lambda}}, \frac{\gamma}{\sqrt{\lambda}}-C \lambda^{-\left(1+\alpha_{0}\right) / 2}<x_{n}<\frac{\gamma}{\sqrt{\lambda}}+C \lambda^{-\left(1+\alpha_{0}\right) / 2}\right\} .
$$

Let $\Omega_{\lambda}=\{x ; x / \sqrt{\lambda} \in \Omega\}, v(x)=u(x / \sqrt{\lambda})\left(x \in \Omega_{\lambda}\right)$ and denote the free boundary of $v$ by $\tilde{\Gamma}_{\lambda}$. Thus $\Delta v=f(v)$ in $\Omega_{\lambda}$ with $\left\{\bar{B}_{\gamma}(0) \times\left(\gamma / 2<x_{n}<3 \gamma / 2\right)\right\} \subset \Omega_{\lambda}$. Moreover

$$
\tilde{\Gamma}_{\gamma} \cap\left\{x ;\left|x^{\prime}\right|<\frac{\gamma}{2},-2 \gamma<x_{n}<2 \gamma\right\}
$$

is contained in $\left\{x ;\left|x^{\prime}\right|<\gamma,\left|x_{n}-\gamma\right| \leqslant C \lambda^{-\alpha_{0} / 2}\right\}$.

Let $x^{\prime} \in \bar{B}_{\gamma / 4}(0)$. Then there is clearly at least one value $\tilde{x}_{n}$ with $\left|\tilde{x}_{n}-\gamma\right|<C \lambda^{-\alpha_{0} / 2}$ such that $\left(x^{\prime}, \tilde{x}_{n}\right) \in \tilde{\Gamma}_{\lambda}$. 
Now set $\sigma=\lambda^{-\alpha_{0} /(8 \beta)}$ and $\rho=c \sigma^{\beta}$ where $\beta$ is as in Theorem 3.3. We deduce that, for some $\lambda_{0}>0$, if $\lambda>\lambda_{0}$ then Theorem 3.3 can be applied about each point $\left(x^{\prime}, \tilde{x}_{n}\right)$. We conclude that $\tilde{x}_{n}$ is unique, $\tilde{x}_{n}=g\left(x^{\prime}\right)$,

$$
\tilde{\Gamma}_{\lambda} \cap\left\{\bar{B}_{\gamma / 4}(0) \times(-2 \gamma, 2 \gamma)\right\}=\left\{x ; x_{n}=g\left(x^{\prime}\right),\left|x^{\prime}\right|<\frac{\gamma}{4}\right\},
$$

and

$$
\begin{gathered}
\left|D g\left(x^{\prime}\right)\right| \leqslant C \sigma \quad \text { for } x^{\prime} \in \bar{B}_{\gamma / 4}(0), \\
\left|D g\left(x_{1}^{\prime}\right)-D g\left(x_{2}^{\prime}\right)\right| \leqslant C \sigma^{1-\alpha \beta}\left|x_{1}^{\prime}-x_{2}^{\prime}\right|^{\alpha} \quad \text { if }\left|x_{1}^{\prime}-x_{2}^{\prime}\right| \leqslant c \sigma^{\beta} .
\end{gathered}
$$

Using (3.9) we see that the last estimate remains valid if $\alpha$ is made smaller. Thus we may assume that $\alpha \beta<\frac{1}{2}$.

If $x_{1}^{\prime}, x_{2}^{\prime} \in \bar{B}_{\gamma / 4}(0)$ with $\left|x_{1}^{\prime}-x_{2}^{\prime}\right|>c \sigma^{\beta}$, then, by (3.14),

$$
\left|D g\left(x_{1}^{\prime}\right)-D g\left(x_{2}^{\prime}\right)\right| \leqslant 2 c \sigma^{1 / 2}\left|x_{1}^{\prime}-x_{2}^{\prime}\right|^{\alpha} .
$$

Combining this with (3.15) and (3.14), we obtain

$$
\left|g\left(x^{\prime}\right)-\gamma\right|_{C^{1+\alpha}\left(\bar{\beta}_{\gamma / 4}(0)\right)} \leqslant c \sigma^{1 / 2}=C \lambda^{-\alpha_{0} / 16 \beta} .
$$

The corresponding portion of $\Gamma_{\lambda}$ is given by $x_{n}=z\left(x^{\prime}\right)=\lambda^{-1 / 2} g\left(\lambda^{1 / 2} x^{\prime}\right)$, so that

$$
\begin{gathered}
\left|D z\left(x^{\prime}\right)\right| \leqslant c \sigma=C \lambda^{-\delta_{1}} \quad \text { for } x^{\prime} \in \bar{B}_{\gamma / 4 \sqrt{\lambda}}(0), \\
\left|z\left(x^{\prime}\right)-\frac{\gamma}{\sqrt{\lambda}}\right|_{C^{1+\alpha}\left(\bar{B}_{\gamma / 4 \sqrt{\lambda}(0))}\right.} \leqslant C \sigma^{1 / 2} \lambda^{\alpha / 2} .
\end{gathered}
$$

Decreasing $\alpha$ again so that $8 \beta \alpha \leqslant \alpha_{0}$, we obtain

$$
\left|z\left(x^{\prime}\right)\right|_{C^{1+\alpha}\left(\bar{B}_{\gamma / 4} \sqrt{\lambda}(0)\right)} \leqslant C .
$$

We now express $z$ in terms of $t$. Write $\nu(t)=\left(\bar{\nu}(t), \nu_{n}(t)\right)$ with $\bar{\nu}(t)=$ $\left(\nu_{1}(t), \ldots, \nu_{n-1}(t)\right)$. We claim that for $t \in \bar{B}_{\gamma /(8 \sqrt{\lambda})}(0)$ the function $k(t, \lambda)$ is well defined. Indeed, if $\left(x_{i}^{\prime}, z\left(x_{i}^{\prime}\right)\right)=h(t)+s_{i} \nu(t)(i=1,2)$ for $s_{1} \neq s_{2}$ then

$$
\frac{\left|z\left(x_{2}^{\prime}\right)-z\left(x_{1}^{\prime}\right)\right|}{\left|x_{2}^{\prime}-x_{1}^{\prime}\right|}=\frac{\left|v_{n}(t)\right|}{|\bar{\nu}(t)|} \text {. }
$$

Since $\nu_{n}(t) \approx 1$ and $|\vec{\nu}(t)| \ll 1$, this contradicts (3.16).

We can now write

$$
\left(x^{\prime}, z\left(x^{\prime}\right)\right)=\left(t+k(t, \lambda) \bar{\nu}(t), \bar{h}(t)+k(t, \lambda) \nu_{n}(t)\right),
$$

so that

$$
\begin{aligned}
x^{\prime} & =t+k(t, \lambda) \bar{\nu}(t), \\
z\left(x^{\prime}\right) & =\bar{h}(t)+k(t, \lambda) \nu_{n}(t) .
\end{aligned}
$$

Since $v_{n}(0)=1$ we can substitute $k(t, \lambda)$ from (3.20) into (3.19) and thus deduce that

$$
x^{\prime}=t+\frac{z\left(x^{\prime}\right)-\bar{h}(t)}{\nu_{n}(t)} \bar{\nu}(t)
$$


from which we conclude (since $\bar{\nu}(0)=0$ ) that $x^{\prime}=\phi(t)$ with $\phi \in c^{1+\alpha}$. Using this in (3.20) we can estimate the $C^{1+\alpha}$ norm of $k(t, \lambda)$, and thus deduce that

$$
|k(t, \lambda)|_{C^{1+\alpha}\left(\bar{B}_{\theta \gamma /} \downarrow \lambda(0)\right)} \leqslant C \quad(\theta>0) .
$$

Moreover, using (3.16),

$$
\left|D_{t} k(t, \lambda)\right|_{0} \leqslant C \lambda^{-\delta_{1}} \quad \text { for } t \in \bar{B}_{\theta \gamma / \sqrt{\lambda}}(0)
$$

In deriving (3.21) we have assumed that (3.12) holds. The first assumption in (3.12) is not restrictive since it can be achieved by renaming the variables $x_{i}$ as $t_{j}$. Next we can perform an affine transformation $t \rightarrow \tau$ in order to satisfy the restrictions on $\bar{h}$ in the new coordinates. This affine transformation is dilatation in the tangential variables but preserves distances along the normals (up to an error term). After deriving (3.21) for $k=k(\tau, \lambda)$ we return to the original coordinates $t$.

Notice that (3.10) is a consequence of (3.16).

Finally, to prove (3.11) it suffices (in view of (3.22)) to estimate

$$
\left|k_{t}(t, \lambda)-k_{t}\left(t_{0}, \lambda\right)\right| \text { when }\left|t-t_{0}\right|>C \lambda^{-1 / 2} \text {. }
$$

Using (3.10) this expression is estimated by $C \lambda^{-\delta_{1}} \leqslant C\left|t-t_{0}\right|^{\delta}$ with suitable $\delta>0$. We can take $\delta_{2}$ in (3.11) as the minimum of $\alpha$ in (3.21) and $\delta$ above.

4. Domains whose graphs have nonnegative mean curvature. For any $x \in \partial \Omega$ denote by $K(x)$ the mean curvature of $\partial \Omega$ at $x$. In this section we assume that

$$
K(x) \geqslant 0 \text { for all } x \in \partial \Omega
$$

this is true, for instance, if $\Omega$ is convex. From Lemma 3.2 it then follows that

$$
|\nabla u|^{2} \leqslant 2 F(u) \text { in } \Omega
$$

where $u$ is any solution of (1.2).

The function $g(u)=\int_{0}^{u} d s / \sqrt{2 F(s)}$ will play a fundamental role in the sequence. Setting $w_{i}=\partial w / \partial x_{i}, w_{i j}=\partial^{2} w / \partial x_{i} \partial x_{j}$ etc., we compute, in $\{u>0\}$,

$$
\begin{gathered}
(g(u(x)))_{i}=g^{\prime}(u) u_{i}, \quad(g(u(x)))_{i i}=g^{\prime \prime}(u) u_{i}^{2}+g^{\prime}(u) f(u), \\
g^{\prime}(u)=\frac{1}{\sqrt{2 F(u)}}, \quad g^{\prime \prime}(u)=-f(u)(2 F(u))^{-3 / 2} .
\end{gathered}
$$

Hence

$$
\Delta g(u)=l(u) \frac{1-|\nabla g(u)|^{2}}{g(u)}
$$

where

$$
l(u)=\frac{f(u) g(u)}{\sqrt{2 F(u)}}=\frac{f(u)}{\sqrt{2 F(u)}} \int_{0}^{u} \frac{d s}{\sqrt{2 F(s)}} .
$$

Notice that $l(t)$ is a positive $C^{1}$ function of $t$ away from $t=0$, whereas near $t=0$ $l(t) \sim$ const $>0$, since $f(u) \sim u^{p}$.

By (4.2),

$$
|\nabla g(u)| \leqslant 1
$$


and thus

$$
\Delta g(u) \geqslant 0 \text { in }\{u>0\} .
$$

We shall need the Hausdorff measure estimate of [1] which is valid for any solution $u$ of (1.2):

$$
\Delta g(u)=d \lambda+I_{\{u>0\}} l(u) \frac{1-|\nabla g(u)|^{2}}{g(u)}
$$

where $d \lambda$ is absolutely continuous with respect to $d H^{n-1} L \Gamma$ ( $\Gamma$ is the free boundary); more precisely,

$$
\begin{gathered}
d \lambda=d H^{n-1} L \Gamma_{\text {red }}+\theta(x) d H^{n-1} L \Gamma_{\text {sing }}, \\
I_{\{u>0\}} l(u) \frac{1-|\nabla g(u)|^{2}}{g(u)} \in L^{1}(\Omega), \quad 0 \leqslant \theta(x) \leqslant \text { const },
\end{gathered}
$$

where $I_{A}$ denotes the characteristic function of a set $A$, and " $\mathrm{L}$ " means "restriction to."

Observation. If $\Gamma$ is smooth and $x_{0} \in \Gamma$ then by Green's formula,

$$
\begin{aligned}
\int_{\{u>0\} \cap B_{R}\left(x_{0}\right)} \Delta g(u)= & \int_{\Gamma \cap B_{R}\left(x_{0}\right)} \nabla g(u) \cdot \nu d H^{n-1} \\
& +\int_{\{u>0\} \cap \partial B_{R}\left(x_{0}\right)} \nabla g(u) \cdot \nu d H^{n-1} .
\end{aligned}
$$

Using (4.5), (4.6) and the relation $|\nabla g(u(x))| \rightarrow 1$ if $\operatorname{dist}(x, \Gamma) \rightarrow 0$ (which is implicit in $(4.8))$, we obtain

$$
H^{n-1}\left(\Gamma \cap B_{R}\left(x_{0}\right)\right) \leqslant H^{n-1}\left[\{u>0\} \cap \partial B_{R}\left(x_{0}\right)\right] .
$$

This estimate is equivalent to $\Gamma$ having nonnegative curvature and, if $n=2$, to $\Gamma$ being convex. In this and in the next section we shall make this observation rigorous, exploiting (4.8) and some elementary facts from geometric measure theory, as well as Theorem 3.4 (for proving that $\Gamma$ is convex if $n=2$ ).

LEMMA 4.1. Let $G$ be any subdomain of $\Omega$ with piecewise smooth boundary $\partial G \subset \Omega$ and with $H^{n-1}(\Gamma \cap \partial G)=0$. Then

$$
\int_{G} \Delta g(u)=\int_{\partial G \cap\{u>0\}} \nabla g(u) \cdot \nu d H^{n-1} .
$$

Proof. This is just Green's formula for a function $g(u(x))$ whose Laplacian is a measure; we shall establish it by approximation.

Let $g(u)_{\varepsilon}$ be a mollification of $g(u(x))$. Then, since $\Delta g$ is a measure,

$$
\Delta\left(g(u)_{\varepsilon}\right) \rightarrow \Delta g(u) \text { as measures. }
$$

Recalling that $(\Delta g(u))(\partial G)=0$ (by (4.7), (4.8), and the assumption $H^{n-1}(\Gamma \cap \partial G)$ $=0)$, we deduce from $(4.10)$ that

$$
\int_{G} \Delta\left(g(u)_{\varepsilon}\right) \rightarrow \int_{G} \Delta g(u) .
$$


By Green's formula for smooth functions we have

$$
\int_{G} \Delta\left(g(u)_{\varepsilon}\right)=\int_{\partial G} \nabla\left(g(u)_{\varepsilon}\right) \cdot \nu d H^{n-1} .
$$

For any small $\delta>0$, let $K$ be any open neighborhood of $\Gamma \cap \partial G$ with $\int_{K \cap \partial G} d H^{n-1}<\delta$. On $(\partial G \backslash K) \cap\{u>0\}$ we have $\nabla\left(g(u)_{\varepsilon}\right) \rightarrow \nabla g(u)$ uniformly as $\varepsilon \rightarrow 0$. On the other hand,

$$
(\partial G \backslash K) \cap\{u=0\} \text { is compactly contained in } \operatorname{int}\{u=0\} ;
$$

hence, if $\varepsilon$ is small enough,

$$
\nabla\left(g(u)_{\varepsilon}\right)=0 \quad \text { on }(\partial G \backslash K) \cap\{u=0\} .
$$

From (4.5) we deduce that $\left|\nabla\left(g(u)_{\varepsilon}\right)\right| \leqslant 1$ and, therefore, if $\varepsilon$ is small enough,

$$
\left|\int_{K \cap \partial G} \nabla\left(g(u)_{\varepsilon}\right) \cdot \nu d H^{n-1}\right|<\delta .
$$

We now break the integral on the right-hand side of (4.12) into $\partial G \cap K$ and $\partial G \backslash K$ and use the preceding remarks; we obtain, letting $\varepsilon \rightarrow 0$ and then $\delta \rightarrow 0$,

$$
\int_{\partial G} \nabla\left(g(u)_{\varepsilon}\right) \cdot \nu d H^{n-1} \rightarrow \int_{\partial G \cap\{u>0\}} \nabla g(u) \cdot d H^{n-1} .
$$

Taking $\varepsilon \rightarrow 0$ in (4.12) and using (4.11), (4.13), the assertion (4.9) follows.

Combining (4.9) with (4.7), (4.8) we have

Corollary 4.2. If $G$ is as in Lemma 4.1, then

$$
\begin{aligned}
\int_{G \cap \Gamma_{\mathrm{red}}} d H^{n-1} & +\int_{G \cap \Gamma_{\text {sing }}} \theta d H^{n-1} \\
& =-\int_{G \cap\{u>0\}} \Delta g(u) d x+\int_{\partial G \cap\{u>0\}} \nabla g(u) \cdot \nu d H^{n-1} .
\end{aligned}
$$

THEOREM 4.3. Every $C^{2}$ portion of $\Gamma$ has nonnegative mean curvature.

Proof. Suppose a smooth portion of $\Gamma$ is given by $x_{n}=h\left(x^{\prime}\right), x^{\prime}=\left(x_{1}, \ldots, x_{n-1}\right)$ varies in a ball $\bar{B}_{R^{\prime}}$, and $u>0$ if $x_{n}<h\left(x^{\prime}\right)$. Since

$$
\Delta g(u) \geqslant 0 \quad \text { in }\{u>0\}, \quad|\nabla g(u)| \leqslant 1,
$$

(4.14) gives

$$
\int_{G \cap \Gamma} d H^{n-1} \leqslant \int_{\partial G \cap\{u>0\}} d H^{n-1}
$$

Take $\partial G \cap\left\{x_{n}<h\left(x^{\prime}\right)\right\}$ to be $x_{n}=h\left(x^{\prime}\right)-\varepsilon \zeta\left(x^{\prime}\right), \zeta \geqslant 0, \zeta \in C_{0}^{2}\left(\bar{B}_{R}\right), \varepsilon>0$, where $\bar{B}_{R}$ is the ball $\left\{\left|x^{\prime}\right|<R\right\}$. Then (4.16) yields

$$
\int_{\bar{B}_{R}} \sqrt{1+|\nabla h|^{2}} \leqslant \int_{\bar{B}_{R}} \sqrt{1+|\nabla h-\varepsilon \nabla \zeta|^{2}} .
$$

From this we deduce that $\nabla \cdot \nabla h / \sqrt{1+|\nabla h|^{2}} \geqslant 0$, and the assertion follows. 
Let $P$ be an open half space with $H^{n-1}(\partial P \cap \Gamma)=0$ and denote by $\bar{\nu}$ the outward normal along $\partial P$. Let $G$ be a convex domain in $P$ with piecewise smooth boundary, such that $\partial G \cap \partial P \neq \phi, u=0$ in an open neighborhood of $\partial G \cap P$.

LEMMA 4.4. Under the foregoing assumptions,

$$
\int_{\{u>0\} \cap \partial G \cap \partial P} d H^{n-1} \leqslant \int_{\Gamma_{\text {red }} \cap G} d H^{n-1}
$$

with strict inequality if $L^{n}(\{u>0\} \cap G)>0$.

Proof. By Green's formula $[8,4.5 .6]$

$$
0=\int_{\{u>0\} \cap G} \operatorname{div} \bar{\nu} d x=\int_{\Gamma_{\mathrm{red}} \cap G} \bar{\nu} \cdot \nu d H^{n-1}+\int_{\{u>0\} \cap \partial G \cap \partial P} \bar{\nu} \cdot \nu .
$$

Hence

$$
\int_{\{u>0\} \cap \partial G \cap \partial P} d H^{n-1}=-\bar{\nu} \cdot \int_{\Gamma_{\mathrm{red}} \cap G} \nu d H^{n-1} \equiv-\bar{\nu} \cdot \nu^{*} .
$$

This gives (4.17). Further, if $L^{n}(\{u>0\} \cap G)>0$, then by an isoperimetric inequality [9] $\nu^{*} \neq 0$ and then the normal at each reduced boundary point is in the direction $\bar{\nu}$. It follows by [9, Theorem 4.8] that $\Gamma \cap G$ is a plane normal to $\bar{\nu}$, which is impossible.

5. Two-dimensional convex domains. In this section we shall study the dead core $N_{u}$ of a solution $u$, assuming that

$$
\Omega \text { is a two-dimensional convex domain. }
$$

By a component of $N_{u}$ we mean a maximal connected subset of $N_{u}$; it is necessarily a closed set.

THEOREM 5.1. If $T$ is a component of $N_{u}$ with nonempty interior, then $T$ is a closed convex domain with $C^{1+\beta}$ boundary, and

$$
\operatorname{dist}\left(T, N_{u} \backslash T\right)>0 .
$$

Proof. Let $O$ be an interior point of $T$ and let $l_{1}, l_{2}$ be rays initiating at $O$ and forming an angle $<\pi$. These rays intersect $\partial T$ for the first time, say, at $P_{1}, P_{2}$. We form the triangle $G=\left\{P_{1}, P_{2}, O\right\}$ and claim that

$$
\{u>0\} \cap G=\varnothing \text {. }
$$

Indeed, otherwise Lemma 4.4 gives (assuming first that $H^{n-1}\left(\overline{P_{1} P_{2}} \cap \Gamma\right)=0$ )

$$
\int_{\overline{P_{1} P_{2}} \cap\{u>0\}} d H^{1}<\int_{\Gamma_{\mathrm{red}} \cap G} d H^{1}
$$

On the other hand, from Corollary 4.2 and (4.15), we obtain $\int_{G \cap \Gamma_{\mathrm{red}}} d H^{1} \leqslant$ $\int_{\partial G \cap\{u>0\}} d H^{1}$, a contradiction.

If $H^{n-1}\left(\overline{P_{1} P_{2}} \cap \Gamma\right)>0$ then since $H_{\text {loc }}^{n-1}(\Gamma)<\infty$ we can find $\tilde{P}_{1}, \tilde{P}_{2}$ with $\tilde{P}_{i} \in \overline{O P_{i}}$ and $\left|\tilde{P}_{i}-P_{i}\right|$ arbitrarily small so that $H^{n-1}\left(\tilde{P}_{1} \tilde{P}_{2} \cap \Gamma\right)=0$ and $\left\{\tilde{P}_{1}, \tilde{P}_{2}, O\right\}$ still violates (5.3) (since $\{u>0\}$ is open). The previous argument can then be applied to $\left\{\tilde{P}_{1}, \tilde{P}_{2}, O\right\}$ in order to derive a contradiction. 
Having proved (5.3), we now denote by $T_{0}$ the union of segments $\overline{O P_{1}}$ when $l_{1}$ varies over all possible directions. From (5.3) it follows that $T_{0}$ is convex; in particular, $\partial T_{0}$ is Lipschitz continuous. Since $n=2$ we can apply Theorem 3.3(b). It then follows that $\partial T_{0}$ is in $C^{1+\beta}$ and $u>0$ in some $\left(\Omega \backslash T_{0}\right)$-neighborhood of $\partial T_{0}$. Hence $T=T_{0}$ and (5.2) holds.

COROLlary 5.2. If $\lambda$ is sufficiently large then $N_{\lambda}$ is a closed convex domain with $C^{1+\beta}$ boundary.

Indeed, by Theorem $3.4, N_{\lambda}$ is a closed domain with $C^{1+\beta}$ boundary; now apply Theorem 5.1.

THEOREM 5.3. Let (2.1) and (5.1) hold. Then the null set $N_{u}$ of the solution of (1.2) is either a closed convex domain with $C^{1+\beta}$ boundary, or a single point, or empty.

Proof. The proof is by continuity with respect to the parameter $\lambda$. For any $\lambda$ there is a unique solution $u_{\lambda}$ (Theorem 2.1) and, by Theorem 1.7, $u_{\lambda}$ increases and

$N_{\lambda}$ decreases as $\lambda$ decreases.

Further, if $\lambda_{n} \rightarrow \lambda$ then $u_{\lambda_{\mu}}(x) \rightarrow u_{\lambda}(x)$ uniformly with respect to $x \in \Omega$.

By Corollary 5.2,

$N_{\lambda}$ is a closed convex domain with $C^{1+\beta}$ boundary, provided $\lambda$ is large enough.

By (5.4) and Theorem 2.2 there is a number $\lambda_{*}>0$ such that

$$
\text { int } N_{\lambda} \neq \varnothing \quad \text { if } \lambda>\lambda_{*}, \quad \text { int } N_{\lambda}=\varnothing \quad \text { if } \lambda<\lambda_{*} .
$$

The continuity $\lambda \rightarrow u_{\lambda}(x)$ implies that $N_{\lambda} \supset \lim \sup _{\lambda_{n} \rightarrow \lambda} N_{\lambda_{n}}$. Recalling (5.4) we then deduce that, whenever $\lambda_{n} \downarrow \lambda$,

$$
N_{\lambda}=\lim _{\lambda_{n} \downarrow \lambda} N_{\lambda_{n}}=\bigcap_{\lambda_{n}} N_{\lambda_{n}}
$$

notice that if each $N_{\lambda_{n}}$ is a closed convex set then the same is true of $\cap N_{\lambda_{n}}$, i.e., $N_{\lambda}$ is also a closed convex set.

We next prove that

if $N_{\lambda_{0}}$ is a closed convex domain then $N_{\lambda}$ is also a closed convex domain for all $\lambda_{0}-\eta<\lambda<\lambda_{0}$, provided $\eta$ is small enough.

Once this is proved, we conclude, upon recalling (5.5) and (5.7), (5.6) that $N_{\lambda}$ is a closed convex domain with $C^{1+\beta}$ boundary if and only if $\lambda>\lambda_{*}$.

To prove (5.8), let $N_{\lambda_{0}, \delta}=\left\{x \in N_{\lambda_{0}}\right.$; $\left.\operatorname{dist}\left(x, \partial N_{\lambda_{0}}\right)>\delta\right\}$. If $u_{\lambda}\left(x^{0}\right)>0$ for some $x^{0} \in N_{\lambda_{0}}$, then, by nondegeneracy $[15,16] \sup _{B_{\delta}\left(x^{0}\right)} u_{\lambda} \geqslant c \delta^{2 /(1-p)}$ provided $\delta \leqslant r_{0}$, where $r_{0}, c$ are small positive constants independent of $\lambda, x^{0}$. Hence, if $\lambda_{0}-\eta<\lambda$ $<\lambda_{0}$ and $\eta$ is small enough depending on $\delta$, say $\eta \leqslant \eta(\delta)$, then

$$
\sup _{B_{\delta}\left(x^{0}\right)} u_{\lambda_{0}} \geqslant \frac{c}{2} \delta^{2 /(1-p)}>0,
$$


and consequently $x^{0} \notin N_{\lambda_{0}, \delta}$. We have thus proved that $N_{\lambda} \supset N_{\lambda_{0}, \delta}$ if $\lambda_{0}-\eta(\delta)<\lambda$ $<\lambda_{0}$. This implies that $\Gamma_{\lambda}$ lies in the region $N_{\lambda_{0}} \backslash N_{\lambda_{0}, \delta}$ of "width" $\delta$ and, consequently (recalling that $\partial N_{\lambda_{0}}$ is in $C^{1+\beta}$, by Theorem 3.3), the flatness condition holds along $\Gamma_{\lambda}$ provided $\delta$ is small enough. Consequently, by Theorem $3.3, \Gamma_{\lambda}$ is a locally $C^{1+\beta}$ graph that can be parametrized by $\Gamma_{\lambda_{0}}$ and, then, by Theorem $5.1 N_{\lambda}$ is a closed convex domain. We have thus completed the proof of (5.8).

It remains to show that $N_{\lambda_{*}}$ consists of a single point; Theorem 1.6 then implies that $N_{\lambda}=\varnothing$ if $\lambda<\lambda_{*}$. Since $N_{\lambda_{*}}=\bigcap_{\lambda>\lambda_{*}} N_{\lambda}, N_{\lambda_{*}}$ is closed convex set. Thus it consists either of a single point or of an interval $I$. We shall assume the latter and derive a contradiction. For simplicity we take $I=\left\{\left(x_{1}, 0\right) ;-a<x_{1}<a\right\}$. Set

$$
\tilde{u}= \begin{cases}u & \text { in } B_{a}^{+}=B_{a} \cap\left\{x_{2} \geqslant 0\right\}, \\ 0 & \text { in } B_{a}^{-},\end{cases}
$$

and notice that $\tilde{u} \in C^{2}$. The free boundary of $\tilde{u}$ is $I$. By [1], the flatness of the free boundary implies that $g(\tilde{u})$ is in $C^{1+\beta}$ in $B_{a}^{+}$, and $|\nabla g(\tilde{u})|=1$ on $x_{2}=0+$.

Consider now the subharmonic function $w=g(\tilde{u})-x_{2}$ in $B_{a}^{+}$. Since $w\left(x_{1}, 0\right)=0$ and $|\nabla g| \leqslant 1, w \leqslant 0$ in $B_{a}^{+}$. Hence, by the maximum principle, either (i) $w \equiv 0$ or (ii) $\partial w\left(x_{1}, 0\right) / \partial x_{2}<0$. The second case implies $\partial g(\tilde{u}) / \partial x_{2}<1$ at $\left(x_{1}, 0\right)$, a contradiction; hence $g(\tilde{u})-x_{2} \equiv 0$ in $B_{a}^{+}$.

In $\Omega_{+}=\Omega \cap\left\{x_{2}>0\right\}$ we can write (4.3) in the form $\Delta g=k(g)\left(1-|\nabla g|^{2}\right)$. Since also $\Delta x_{2}=0=k\left(x_{2}\right)\left(1-\left|\nabla x_{2}\right|^{2}\right)$, we can write $\Delta w=A \cdot \nabla w+$ ew in $\Omega_{+}$, with smooth coefficients $A, e$. Hence, by unique continuation, $w \equiv 0$ in $\Omega_{+}$, which is a contradiction since $w=g(1)-x_{2}$ on $\partial \Omega \cap\left\{x_{2}>0\right\}$.

REMARK 5.1. Consider $\Delta w=h(w)$ in $\Omega \backslash K, w=0$ on $\partial \Omega, w=1$ on $\partial K$, where $\Omega$ and $K$ are convex domains in $R^{n}$ and $\bar{K} \subset \Omega$. Caffarelli and Spruck [4] proved that if

$$
h(t) \geqslant 0, \quad h^{\prime}(t) \geqslant 0 \quad \forall t, h(0)=0,
$$

then the sets $\bar{K} \cup\{w>c\}$ are convex for all $c \in(0,1)$. If we set $w=1-u$ in (1.2), then (1.2) reduces to

$$
\Delta w=h(w) \text { in } \Omega, \quad w=0 \text { on } \partial \Omega,
$$

with $w=1$ in the dead core, where $h(t)=-f(1-t)$. The assumptions on $f$ made in Theorem 5.1 are, in terms of $h$ :

$$
\begin{aligned}
& h(t)<0, \quad h^{\prime}(t)+|h(t)| / t>0 \quad \text { for } 0 \leqslant t<1, \\
& h(t) \sim-c(1-t)^{p} \quad \text { as } t \uparrow 1(c>0), \quad h(t)=0 \quad \text { if } t>1 .
\end{aligned}
$$

The basic difference in the respective assumptions on $h$ is in the signature of $h$.

REMARK 5.2. Denote by $\Sigma$ the set of $\lambda$ 's for which the variational problem $\left(J_{\lambda f}\right)$ does not have a unique solution. We claim that

$$
\Sigma \text { is countable. }
$$

Indeed, for any large $A>0$ choose $x^{0} \in \Omega, x^{0}$ near $\partial \Omega$ such that $u_{\lambda}\left(x^{0}\right)>0$ if $0<\lambda<A$, where $u_{\lambda}$ is a minimizer of $\left(J_{\lambda f}\right)$. By Theorem 1.7, if $\lambda<\lambda^{\prime}<A$ then $u_{\lambda}\left(x^{0}\right)>u_{\lambda^{\prime}}\left(x^{0}\right)$. By Theorem 1.4, if $u_{\lambda}, v_{\lambda}$ are two distinct minimizers for $J_{\lambda f}$ then $u_{\lambda}\left(x^{0}\right) \neq v_{\lambda}\left(x^{0}\right)$. We conclude that there is a monotone graph $\lambda \rightarrow \Phi(\lambda)(0<\lambda<A)$ 
such that $\Phi(\lambda)$ is a real number in $(0,1)$ if $\lambda \notin \Sigma$ and $\Phi(\lambda)$ is a nonzero interval if $\lambda \in \Sigma$. The assertion (5.9) now readily follows.

In the next result we drop the condition (2.1).

THEOREM 5.4. Let $0<\lambda_{0}<\lambda_{1}<\infty$ and suppose that for $\lambda \in\left[\lambda_{0}, \lambda_{1}\right), \lambda \rightarrow u_{\lambda}$ is $a$ family of solutions of (0.1) which varies continuously with $\lambda$ (i.e, $\lambda \rightarrow u_{\lambda}(x)$ is continuous for each $x \in \Omega$ ) and $u_{\lambda} \leqslant u_{\lambda^{\prime}}$ if $\lambda \geqslant \lambda^{\prime}$. Then (i) if $K_{\lambda_{0,1}, 1}$ and $K_{\lambda_{0}, 2}$ are distinct components of $N_{\lambda_{0}}$ then they are contained in distinct components of $N_{\lambda}$ for $\lambda_{0}<\lambda<\lambda_{1}$; (ii) each component of $N_{\lambda_{0}}$ is either a point or a closed convex domain; (iii) the number of components of $N_{\lambda_{0}}$ is finite.

If for any $\lambda \in\left(\lambda_{0}, \lambda_{1}\right)$ there is a unique minimizer $u_{\lambda}$, then $u_{\lambda}$ satisfies the assumptions of Theorem 5.4. Indeed the monotonicity follows from Theorem 1.7, and the continuity in $\lambda$ of $u_{\lambda}$ is assured by the uniqueness. Another natural candidate is the family of minimal (or maximal) solutions; here one must first establish their continuous dependence upon $\lambda$.

Proof. From the monotonicity assumptions and Theorem 1.6, $K_{\lambda_{0}, 1} \subset$ int $N_{\lambda}$ for $\lambda_{0}<\lambda$. Hence $K_{\lambda_{0}, 1} \subset$ int $K_{\lambda}$ where $K_{\lambda}$ is a component of $N_{\lambda}$. Since int $K_{\lambda} \neq \varnothing$ it follows from Theorem 5.1 that $K_{\lambda}$ is a closed convex domain. Using the monotonicity and continuity assumptions on $u_{\lambda}$ we can argue as in the proof of Theorem 5.3 to conclude that $N_{\lambda_{0}} \cap K_{\lambda}$ is either a point or a closed convex domain. In particular, it is connected. Hence $\left(N_{\lambda_{0}} \backslash K_{\lambda_{0.1}}\right) \cap K_{\lambda}=\varnothing$, which proves the assertion (i). Thus we have $N_{\lambda_{0}} \cap K_{\lambda}=K_{\lambda_{0.1}}$ and from the above it follows that this is either a point or a closed convex domain. Hence we have the second assertion.

Finally suppose that $N_{\lambda_{0}}$ has an infinite number of components $K_{i}$. Then there exists a sequence of points $X_{i} \in K_{i}$ such that $X_{i} \rightarrow X_{0} \in K_{0}$, a component of $N_{\lambda_{0}}$.

Since $K_{0} \subset$ int $N_{\lambda}$ for any $\lambda>\lambda_{0}$, there is a component $K_{\lambda}$ of $N_{\lambda}$ with $X_{0} \in K_{0} \subset$ int $K_{\lambda}$. But then we have $K_{i} \cap K_{\lambda} \neq \varnothing$ for $i$ sufficiently large, which contradicts (i).

From Theorem 5.4 we deduce

COROLlary 5.5. For each $\lambda \in\left[\lambda_{0}, \lambda_{1}\right), N_{\lambda}$ has a finite number of components, where each component is either a point or a closed convex domain. If one such component is a point then

$(\alpha)$ it disappears from $N_{\lambda^{\prime}}$ for $\lambda^{\prime}<\lambda$,

( $\beta$ ) it develops into a closed convex domain of $N_{\lambda^{\prime}}$ for $\lambda^{\prime}>\lambda$.

Finally, the number of components is finite and increases as $\lambda$ increases.

6. The coincidence sets for variational inequalities. Consider the variational inequality

$$
\left.\begin{array}{r}
-\Delta u \geqslant 0 \\
u-\phi \geqslant 0 \\
-\phi) \Delta u=0
\end{array}\right\} \quad \text { a.e. in } \Omega,
$$

THEOREM 6.1. If $\Omega$ is a convex set in $R^{2}$ with $C^{2+\alpha}$ boundary $(0<\alpha<1)$ and if $\Delta \phi \equiv-c$ for some positive constant $c$, then the coincidence set $\{u=\phi\}$ is convex. 
A related result was proved by Lewy and Stampacchia [11] (by an entirely different method) namely: If (6.2) is replaced by

$$
u>\phi \quad \text { on } \partial \Omega
$$

and $\Delta \phi \equiv-c$ is replaced by

$\phi$ is strictly concave and smooth,

then

$\{u=\phi\}$ is a simply connected closed domain with Jordan boundary.

Let us show that

the conditions (6.3), (6.4) (or even (6.2), (6.4)) do not yield, in general, the assertion that $\{u=\phi\}$ is convex.

Proof of (6.6). Consider the case where $\phi$ describes a pyramid whose base is a square having diagonals on the $x$ - and $y$-axes. If $\phi=0$ on $\partial \Omega$ and $u=1$ on $\partial \Omega$ then $\{u=\phi\}$ is nonempty provided $\sup _{\Omega} \phi>1$ (otherwise $\Delta u=0$ and $u \equiv 1$ in $\Omega$, so that $u<\phi$ at some point in $\Omega$ ).

The set $\{u=\phi\}$ cannot include points of faces of $\phi$. Indeed, if $u\left(x_{0}\right)=\phi\left(x_{0}\right)$ and $\left(x_{0}, \phi\left(x_{0}\right)\right)$ lies on a face of the pyramid, then $-\Delta(u-\phi)=-\Delta u \geqslant 0$ in a neighborhood $N$ of $x_{0}$ and, since $u-\phi \geqslant 0$, the strong maximum princple gives $u-\phi>0$ in $N$.

We conclude that

$$
\{u=\phi\} \text { consists of points lying on the edges of the pyramid. }
$$

This gives the counterexample asserted in (6.6) in case of a $\phi$ which is not smooth. To get a counterexample for a smooth obstacle we approximate $\phi$ by strictly concave mollifiers $\phi_{j}$ and denote the corresponding solutions by $u_{j}$. By [11], the set $C_{j}=\left\{u_{j}\right.$ $\left.=\phi_{j}\right\}$ is a simply connected closed domain. We assume that

$$
C_{j} \text { is convex for each } j
$$

and derive a contradiction.

By (6.7) $u-\phi>0$ on $\{x= \pm y, y \neq 0\} \cap \bar{\Omega}$. Hence, for any $\varepsilon>0, u_{j}-\phi_{j}>0$ on $\{x= \pm y,|y| \geqslant \varepsilon\} \cap \bar{\Omega}$ provided $j \geqslant j(\varepsilon)$. If (6.8) holds then we conclude that the diameter of $C_{j}$ is $\leqslant \sqrt{2} \varepsilon$. Hence

$$
\Delta(u-\phi)=\lim _{j} \Delta\left(u_{j}-\phi_{j}\right)=0 \quad \text { (weak limit) }
$$

if $x^{2}+y^{2}>4 \varepsilon^{2}$, i.e., $\Delta(u-\phi)=0$ a.e. which contradicts the facts that $u \in C^{1,1}$, $\phi \notin C^{1,1}$.

Proof of Theorem 6.1. Theorem 5.3 holds for $f(t)=c t^{p}$ with $0<p<1$. Going over the details of the proof we find that the proof extends to the (limiting) case $f(t)=c \chi_{[t>0]}$; here the functional $J$ is $J(v)=\int_{\Omega}\left[\frac{1}{2}|\nabla v|^{2}+c v^{+}\right] d x$. Thus the coincidence set for the variational inequality

$$
\left.\begin{array}{rr}
-\Delta U & \geqslant-c \\
U \geqslant & 0 \\
U(-\Delta U+c) & =0
\end{array}\right\} \quad \text { a.e. in } \Omega,
$$




$$
U=1 \text { on } \partial \Omega
$$

is convex. Setting $u=U+\phi$, we have

$$
\begin{array}{ll}
-\Delta u=-\Delta U-\Delta \phi \geqslant-c-\Delta \phi=0 & \text { if } U=0, \\
-\Delta u=-\Delta U-\Delta \phi=-c-\Delta \phi=0 & \text { if } U>0,
\end{array}
$$

and (6.1), (6.2) follow.

COROLlaRY 6.2. Let $\Omega$ be a bounded convex domain in $R^{2}$ with $C^{2+\alpha}$ boundary and denote by $\psi$ the solution of

$$
\Delta \psi=-1 \quad \text { in } \Omega, \quad \psi=0 \quad \text { on } \partial \Omega .
$$

Then there exists a unique point $X^{0} \in \Omega$ such that $\psi\left(X^{0}\right)=\max _{\bar{\Omega}} \psi$.

Proof. Set $M=\max _{\bar{\Omega}} \psi, U=M-\psi$. Then $U$ satisfies (6.9) with $c=1$ and $U=M$ on $\partial \Omega$. It follows that the set $K=\{U=0\}=\{\psi=M\}$ is convex. By analyticity of $\psi, K$ has no interior and thus it consists of either one point (as asserted), or an interval $I$. Suppose the second case occurs and take for simplicity $I \subset\{y=0\}$. Applying the Cauchy-Kowalewsky theorem to $U-y^{2} / 2$ we find that $U-y^{2} / 2 \equiv 0$, a contradiction.

7. The third boundary value problem. In this section we shall extend the main results of the previous sections to the third boundary value problem. Thus we consider

$$
\begin{aligned}
& \Delta u=f(u) \text { in } \Omega, \\
& \frac{\partial u}{\partial \nu}+\mu(u-1)=0 \quad \text { on } \partial \Omega
\end{aligned}
$$

where $f(t)$ is a function satisfying (1.1), $\nu$ is the outward normal, and $\mu$ is a given positive number. We shall imbed this problem in the family of elliptic problems

$$
\begin{aligned}
& \Delta u_{\lambda}=\lambda f\left(u_{\lambda}\right) \text { in } \Omega, \\
& \frac{\partial u_{\lambda}}{\partial \nu}+\mu \sqrt{\lambda}\left(u_{\lambda}-1\right)=0 \text { on } \partial \Omega
\end{aligned}
$$

where $0<\lambda<\infty$.

The existence of a solution of (7.1), (7.2) follows by minimizing the functional

$$
J_{\mu}(v)=\int_{\Omega}\left(\frac{1}{2}|\nabla v|^{2}+F(v)\right)+\mu \int_{\partial \Omega}\left(\frac{1}{2} v^{2}-v\right), \quad v \in H^{1}(\Omega) .
$$

One can also construct minimal solutions and maximal solutions as in $\S 1$.

THEOREM 7.1. For any solution of (7.1), (7.2) there holds

$$
\begin{aligned}
& 0 \leqslant u<1 \quad \text { in } \Omega, \\
& \frac{\partial u}{\partial \nu}>0 \quad \text { on } \partial \Omega .
\end{aligned}
$$

Proof. If the set $K=\{u>0\}$ is nonempty then $\partial K \cap \partial \Omega$ contains an open portion of $\partial \Omega$ (otherwise $u \equiv 0$ in $K$, since $\Delta u=0$ in $K$ ) and $u$ takes in $\bar{K}$ negative minimum at an interior point $X$ of $\partial K \cap \partial \Omega$. But then $\partial u / \partial \nu<0, u-1<0$ at $X$, and (7.2) is contradicted. 
If $u$ takes maximum in $\bar{\Omega}$ at $Y$, and $u(Y) \geqslant 1$ then $Y \in \partial \Omega$ (since $\Delta u \geqslant 0$ in $\Omega$ ) and $\partial u / \partial v>0, u-1 \geqslant 0$ at $Y$, again contradicting (7.2). We have thus completed the proof of (7.4); (7.5) follows from (7.4) and (7.2).

THEOREM 7.2. If $f$ satisfies (2.1) then the solution of (7.1), (7.2) is unique.

Proof. Setting $u=1-w^{\beta}(\beta>0)$ we find that $w$ satisfies (2.2), (2.3), and $\partial w / \partial \nu+\mu / \beta w=0$ on $\partial \Omega$. The uniqueness result of Corollary 1.2 easily extends to the present case.

From now on we assume that

$\Omega$ is a convex domain in $R^{2}$ with $\partial \Omega$ in $C^{3+\alpha}$.

We shall denote points in $R^{2}$ by $X=(x, y)$.

LeMma 7.3. Any solution of (7.1), (7.2) satisfies

$$
|\nabla u|^{2} \leqslant 2 F(u) \text { in } \Omega \text {. }
$$

Proof. Set $\Phi=|\nabla u|^{2}-2 F(u)$. As in [12] $\Phi$ cannot take its maximum in $\bar{\Omega}$ at an interior point, since

$$
\Delta \Phi-\frac{f}{|\nabla u|^{2}} \nabla u \cdot \nabla \Phi \geqslant 0 \quad \text { in }\{\nabla u \neq 0\} .
$$

Thus, if (7.7) is not true then $\Phi$ takes positive maximum at a point $X^{0} \in \partial \Omega$. Take for definiteness $X^{0}=(R, 0)$ with $x=R-\alpha y^{2}+O\left(y^{3}\right) \equiv g(y)$ describing $\partial \Omega$ near $X^{0}$, and the segment $\{(x, 0), 0 \leqslant x<R\}$ in $\Omega$; since $\Omega$ is convex, $\alpha \geqslant 0$.

By (7.5), $u_{x}>0$ at $X^{0}$; further, by the maximum principle, $\Phi_{x}\left(X^{0}\right)>0$, i.e.,

$$
u_{x} u_{x x}+u_{y} u_{x y}-f u_{x}>0 \text { at } X^{0} .
$$

The boundary condition (7.2) can be written in the form

$$
u_{x}(g(y), y)-g^{\prime}(y) u_{y}(g(y), y)+\mu(u-1) \sqrt{1+\left(g^{\prime}(y)\right)^{2}}=0 .
$$

Since $\Phi(g(y), y)$ takes maximum at $y=0, d \Phi / d y=0, d^{2} \Phi / d y^{2} \leqslant 0$ at $y=0$, i.e,

$$
\begin{gathered}
\Phi_{y}=0 \text { at } X^{0}, \\
-2 \alpha \Phi_{x}+\Phi_{y y} \leqslant 0 \text { at } X^{0} .
\end{gathered}
$$

Differentiating (7.9) and taking $y=0$, we get $u_{x y}+2 \alpha u_{y}+\mu u_{y}=0$, that is,

$$
u_{x y}=-(2 \alpha+\mu) u_{y} \text {. }
$$

Next, (7.10) can be written in the form $u_{x y} u_{x}+u_{y y} u_{y}-f u_{y}=0$, so that, after using (7.12) and (7.1),

$$
u_{y}\left[(2 \alpha+\mu) u_{x}+u_{x x}\right]=0 \text { at } X^{0} .
$$

Consider first the case $u_{y}\left(X^{0}\right) \neq 0$. Then we get from (7.13), $u_{x x}=-(2 \alpha+\mu) u_{x}$ $<0$; hence $u_{x} u_{x x}<0$. Since, further $u_{y} u_{x y}<0$ by (7.12), the left-hand side of (7.8) is negative, a contradiction.

Consider next the case $u_{y}\left(X^{0}\right)=0$. Then, by (7.12), also $u_{x y}\left(X^{0}\right)=0$ and, by (7.8), $u_{x x}-f>0, u_{y y}<0$ at $X^{0}$. 
Differentiating (7.9) twice and substituting $y=0$ we then obtain

$$
-2 \alpha u_{x x}+u_{x y y}+4 \alpha u_{y y}+4 \alpha^{2} \mu(u-1)=0 \text { at } X^{0} .
$$

From (7.11) we also obtain

$$
-2 \alpha\left(u_{x x} u_{x}-f u_{x}\right)+u_{x} u_{x y y}+u_{y y}^{2}-f u_{y y} \leqslant 0
$$

at $X^{0}$. Substituting $u_{x y y}$ from (7.14) into the last inequality, we get

$$
-2 \alpha\left(u_{x x}-f\right)+\frac{u_{y y}^{2}}{u_{x}}-\frac{f u_{y y}}{u_{x}}-2 \alpha u_{x x}-4 \alpha u_{y y}+4 \alpha^{2} \mu(1-u) \leqslant 0,
$$

or

$$
2 \alpha f+\frac{u_{y y}^{2}}{u_{x}}-\frac{f u_{y y}}{u_{x}}-4 \alpha u_{y y}+4 \alpha^{2} \mu(1-u) \leqslant 0
$$

which is a contradiction since all the terms on the left are nonnegative (recall that $\alpha \geqslant 0$ ) and the second and third ones are positive.

We shall now study the null sets $N_{\lambda}$. Using Lemma 7.3 we can establish as in $\$ 5$

LEMMA 7.4. If a component of $N_{\lambda}$ has nonempty interior then it is a convex set.

LEMMA 7.5. There exists a positive number $\gamma$ such that

$$
\Omega_{(\gamma+o(1)) / \sqrt{\lambda}} \subset N_{\lambda} \subset \Omega_{(\gamma+o(1)) / \sqrt{\lambda}}
$$

where $o(1) \rightarrow 0$ uniformly as $\lambda \rightarrow \infty$.

Proof. We begin with the crude estimates

$$
\Omega_{c_{0} / \sqrt{\lambda}} \subset N_{\lambda} \subset \Omega_{c / \sqrt{\lambda}} \quad\left(c_{0}>0, c>0\right) .
$$

To prove the left-hand side we can use the same supersolution

$$
v(X)=A\left|X-X_{0}\right|^{2 /(1-p)}
$$

as in Theorem 3.1, since $v(X) \geqslant 1>u_{\lambda}$ on $\partial \Omega$. To prove the right-hand side of (7.16) we set $w_{\lambda}(X)=u_{\lambda}(X / \sqrt{\lambda})$ and $\Omega_{\lambda}=\{X / \sqrt{\lambda} ; X \in \Omega\}$. Since $\partial w_{\lambda} / \partial \nu+$ $\mu\left(w_{\lambda}-1\right)=0$ on $\partial \Omega_{\lambda}$, elliptic estimates give $\left|D w_{\lambda}\right| \leqslant C$, i.e., $\left|D u_{\lambda}\right| \leqslant C \sqrt{\lambda}$. Thus it suffices to establish a uniform lower bound

$$
u_{\lambda} \geqslant c \text { on } \partial \Omega, \quad c>0 \text {. }
$$

If this is not true when we can find sequences $\lambda_{m} \rightarrow \infty, X^{m} \in \partial \Omega$ such that $u_{\lambda_{m}}\left(X^{m}\right) \rightarrow 0, X^{m} \rightarrow \bar{X}$ as $m \rightarrow \infty$. Suppose for simplicity that the tangent to $\partial \Omega$ at $\bar{X}$ is horizontal and set $w_{m}(X)=u_{\lambda_{m}}\left(\left(X-X^{m}\right)\right) / \sqrt{\lambda_{m}}$. Then

$$
\begin{aligned}
& \Delta w_{m}=f\left(w_{m}\right) \quad \text { in } \tilde{\Omega}_{m, R}=\left\{-C<y<\phi_{m}(x),-R<x<R\right\}, \\
& \frac{\partial w_{m}}{\partial \nu}+\mu\left(w_{m}-1\right)=0 \quad \text { on } y=\phi_{m}(x), \quad \phi_{m}(0)=0, \\
& 0 \leqslant w_{m} \leqslant 1 \quad \text { in } \tilde{\Omega}_{m, R},
\end{aligned}
$$

for any $C>0, R>0$ and $m \geqslant m_{0}(C, R)$, and $w_{m}(0,0) \rightarrow 0$ as $m \rightarrow \infty$. Notice that $y=\phi_{m}(x)$ is a translate of $\partial \Omega_{\lambda_{m}}$, and, $\left|D^{j} \phi_{m}(x)\right| \leqslant \eta\left(\lambda_{m}\right) \rightarrow 0$ if $m \rightarrow \infty(0 \leqslant j \leqslant 3)$, uniformly with respect to $x \in(-R, R)$. 
By elliptic estimates we may assume that $w_{m} \rightarrow w$ uniformly in compact sets and

$$
\begin{aligned}
& \Delta w=f(w) \quad \text { in } T=\left\{-\frac{C}{2}<y<0,-\frac{R}{2}<x<\frac{R}{2}\right\}, \\
& \frac{\partial w}{\partial y}+\mu(w-1)=0 \quad \text { on } y=0, \\
& 0 \leqslant w \leqslant 1 \quad \text { in } T .
\end{aligned}
$$

Since $w(0,0)=0$, if follows that $w_{y}+\mu(w-1) \leqslant-\mu<0$ at $(0,0)$, a contradiction to the second equation in (7.18).

Having proved (7.16) we now proceed to establish (7.15). For any point $\bar{X} \in \partial \Omega$ we consider

$$
w_{\lambda}(X)=u_{\lambda}\left(\frac{X-\bar{X}}{\sqrt{\lambda}}\right) \quad \text { in } \tilde{\Omega}_{\lambda}=\left\{\frac{X-\bar{X}}{\sqrt{\lambda}} ; X \in \Omega\right\} .
$$

We shall assume for simplicity that the tangent to $\partial \Omega$ at $\bar{X}$ is horizontal. Notice that the $\partial \Omega$-neighborhood of $\bar{X}$ is mapped into $y=\phi_{\lambda}(x)$ with $\phi_{\lambda}(0)=0$ and for any $R>0$

$$
\left|D^{j} \phi_{\lambda}(x)\right| \leqslant \eta(\lambda) \quad \text { if }|x|<2 R, \quad 0 \leqslant j \leqslant 3
$$

where $\eta(\lambda) \rightarrow 0$ if $\lambda \rightarrow \infty$. From (7.16) and its proof we have, for $\lambda \geqslant \lambda_{0}(R)$,

$$
\begin{aligned}
& w_{\lambda} \geqslant c>0 \quad \text { if }-c_{1} \leqslant y \leqslant 0, \quad|x|<2 R, \\
& w_{\lambda}=0 \quad \text { if }-c_{3} \leqslant y \leqslant-c_{2}, \quad|x|<2 R \text {, }
\end{aligned}
$$

where $0<c_{1}<c_{2}<c_{3}$, and $c_{i}$ are independent of $\lambda, R$. By Lemma 7.4 the component $\tilde{N}_{\lambda}$ of $\left\{w_{\lambda}=0\right\}$ which contains the point $\left(0,-c_{2}\right)$ is convex. Setting

$$
\tilde{\Omega}_{\lambda, R}=\left\{-c_{3}<y<\phi_{\lambda}(x),-R<x<R\right\},
$$

the set $\tilde{\Omega}_{\lambda, R} \cap \tilde{N}_{\lambda}$ is bounded by the lines $\left\{y=-c_{3}\right\},\{x= \pm R\}$ and a curve $y=k_{\lambda}(x)$, where $k_{\lambda}(x)$ is concave for $|x|<2 R$. Since $-c_{2}<k_{\lambda}(x)<-c_{1}$, we deduce that $\left|k_{\lambda}^{\prime}(x)\right| \leqslant C$ a.e. for $|x|<R$. Hence for any subsequence of $\lambda$ 's converging to $\infty$ there is a subsequence for which

$$
k_{\lambda}(x) \rightarrow k(x), \quad w_{\lambda}(X) \rightarrow w(X)
$$

uniformly in compact subsets in $R^{2}$, and $k(x)$ is concave and $-c_{2} \leqslant k(x) \leqslant-c_{1}$. It follows that

$$
k(x) \equiv \text { const }=-\gamma_{0}
$$

Recall also that

$$
\Delta w_{\lambda}=f\left(w_{\lambda}\right) \text { in } \tilde{\Omega}_{\lambda} .
$$

From (7.21)-(7.23) and nondegeneracy [1] it follows that $\left\{y=-\gamma_{0}\right\}$ lies in the free boundary of $w$. Applying Lemma 7.6 below we deduce that $w$ is a 1-dimensional solution $w(x)$. To compute it, we need to solve $\zeta^{\prime}(t)=\sqrt{2 F(\zeta(t))}$ for $t<0$, $\zeta^{\prime}(0)+\mu(\zeta(0)-1)=0 . \zeta(0)$ is determined by $-\mu(\zeta(0)-1)=\sqrt{2 F(\zeta(0))}$, i.e., $\sigma \equiv$ $\zeta(0)$ is the solution of $\chi(\sigma)=0$ where $\chi(x)=\sqrt{2 F(s)}+\mu(s-1)$. Noting that 
$\chi(0)<0, \chi(1)>0, \chi^{\prime}(s)>0$, there is indeed a unique solution $\sigma$. Denote by $\gamma$ the smallest positive number such that $\zeta(-\gamma)=0$. Then $w=\zeta\left(z-\gamma+\gamma_{0}\right)$ and $\gamma_{0}=\gamma$.

Since $w$ is uniquely determined, we conclude that as $\lambda \rightarrow \infty$.

$$
w_{\lambda}(X) \rightarrow \zeta(x) \text {. }
$$

Hence for any $\varepsilon>0$ the free boundary of $w_{\lambda}$ in $\tilde{\Omega}_{\lambda, R}$ lies in the strip $|y+\gamma|<\varepsilon$ provided $\lambda \geqslant \lambda(\varepsilon, R)$. Finally, since $\lambda(\varepsilon, R)$ can be taken uniformly with respect to the initial point $\bar{X}$ on $\partial \Omega$ (otherwise we construct sequences $\bar{X}_{m} \in \partial \Omega$ and $\lambda_{m} \rightarrow \infty$ and derive a contradiction by working with $\left.w_{\lambda_{m}}(X)=u_{\lambda}\left(\left(X-\bar{X}_{m}\right) / \sqrt{\lambda_{m}}\right)\right),(7.15)$ follows.

LEMMA 7.6. Let

$$
T_{\mathfrak{b}, R}=\{-\mathfrak{b}<y<0 ;-R<x<R\} \quad(\mathfrak{b}>0, R>0)
$$

and let $u$ be a solution of

$$
\begin{aligned}
& 0 \leqslant u \leqslant 1, \quad \Delta u=f(u) \quad \text { in } T_{\mathfrak{b}, R}, \\
& u(x, y)=0 \quad \text { if }-R<x<R, \quad-\mathfrak{b} \leqslant y \leqslant-\gamma_{0} \quad\left(\gamma_{0} \in(0, \mathfrak{b})\right), \\
& \left\{\left(x,-\gamma_{0}\right) ;-R<x<R\right\} \subset \partial\{u>0\},
\end{aligned}
$$

satisfying (7.7) in $T_{\mathfrak{b}, R}$. Then $u(x, y) \equiv u(y)$ and $u(y)>0$ if $-\gamma_{0}<\gamma<0$.

Proof. By [8, 2.10.19(4)], for any $h \in L^{1}\left(R^{n}\right), s>0$,

$$
\lim _{r \rightarrow 0} r^{s-n} \int_{B_{r}(y)} h(x) d x=0
$$

except for $y$ in an $H^{n-s}$-null set. Recalling (4.8) and $l(u) \rightarrow$ const $>0$ as $u \rightarrow 0$, we see that $\left[\left(1-|\nabla g(u)|^{2}\right) / g(u)\right] I_{\{u>0\}} \in L^{1}$, so that

(7.26) $\lim _{t \rightarrow 0} \frac{1}{t} \int_{B_{t}\left(\xi,-\gamma_{0}\right) \cap\{u>0\}} \frac{1-|\nabla g(u)|^{2}}{g(u)} d Y=0 \quad$ for a.a. $\xi \in(-R, R)$.

Fix such $\xi$ and consider, for any small $\tau>0$,

$$
v_{\tau}(X)=\frac{1}{\tau} g\left(u\left(\tau X+\left(\xi,-\gamma_{0}\right)\right)\right) \text { in } B_{2}(0) \text {. }
$$

Notice that

$$
\begin{aligned}
v_{\tau}(x, y) & =0 \quad \text { if } y \leqslant 0, \\
\frac{1-\left|\nabla v_{\tau}(X)\right|^{2}}{v_{\tau}(X)} & =\tau \frac{1-|\nabla g(u(Y))|^{2}}{g(u(Y))}
\end{aligned}
$$

where $Y=\tau X+\left(\xi,-\gamma_{0}\right)$. By (7.26),

$$
\begin{aligned}
0 & =\lim _{\tau \rightarrow 0} t^{-1} \int_{B_{2 \tau}\left(\xi,-\gamma_{0}\right) \cap\{u>0\}} \frac{1-|\nabla g(u(Y))|^{2}}{g(u(Y))} d Y \\
& =\lim _{\tau \rightarrow 0} \int_{B_{2}(0) \cap\left\{v_{\tau}>0\right\}} \frac{1-\left|\nabla v_{\tau}(X)\right|^{2}}{v_{\tau}(X)} d X .
\end{aligned}
$$


Since, by $(4.5),\left|\nabla v_{\tau}\right| \leqslant 1$, there is a sequence $\tau_{i} \downarrow 0$ with $v_{\tau_{i}}(X) \rightarrow v_{0}(X)$ uniformly in $B_{1}(0)$ and (7.29) gives (using Fatou's lemma) $\left|\nabla v_{0}\right| \equiv 1$ in $B_{2}(0) \cap\left\{v_{0}>0\right\}$. Since, by (4.3), (7.28), $\left|\Delta v_{\tau}\right| \leqslant C\left(1-\left|\nabla v_{\tau}\right|^{2}\right) / v_{\tau}$, we also have $\Delta v_{0}=0$ in $B_{2}(0) \cap\left\{v_{0}>0\right\}$. By (7.27) $v_{0}(x, y)=0$ if $-2<x<2, y \leqslant 0$, and, by nondegeneracy [1],

$$
(0,0) \in \partial\left\{v_{0}>0\right\} \text {. }
$$

It now easily follows that $v_{0}(x, y)=y^{+}$in $B_{2}(0)$.

Consider the function $z(X)=g(u(X))-\left(y+\gamma_{0}\right)^{+}$in $T_{\mathfrak{b}, R}$. From (4.5), (4.6),

$$
\Delta z \geqslant 0 \text { in } \mathscr{Q}^{\prime}\left(T_{\mathfrak{b}, R}\right) \text {, }
$$

and

$$
\frac{\partial z}{\partial y}=\frac{\partial g(u)}{\partial y}-1 \leqslant 0 \quad \text { in } T_{\mathfrak{b}, R} \cap\left\{y>-\gamma_{0}\right\} .
$$

Also $z\left(x,-\gamma_{0}\right)=0$, so that

$$
z \leqslant 0 \quad \text { in } T_{\mathfrak{b}, R}, \quad z=0 \quad \text { in } T_{\mathfrak{b}, R} \cap\left\{y \leqslant-\gamma_{0}\right\} .
$$

Next for any $\xi$ as in (7.26),

$$
\begin{aligned}
\lim _{\tau_{i} \rightarrow 0} \frac{z\left(\xi,-\gamma_{0}+\tau_{i}\right)}{\tau_{i}} & =\lim _{\tau_{i} \rightarrow 0} \frac{g\left(u\left(\xi,-\gamma_{0}+\tau_{i}\right)\right)}{\tau_{i}}-1 \\
& =\lim _{\tau_{i} \rightarrow 0} v_{\tau_{i}}(0,1)-1=v_{0}(0,1)-1=0 .
\end{aligned}
$$

Thus $\partial z / \partial y=0$ at $\left(\xi,-\gamma_{0}\right)$. Recalling (7.30) and using the strong maximum principle, we deduce that $z \equiv 0$, i.e., $g(u(x, y))=\left(y+\gamma_{0}\right)^{+}$, and the assertion follows.

We shall need the following comparison result.

LEMMA 7.7. If (2.1) holds and

$$
f^{\prime}(t)+\frac{f(t)}{t}>0 \quad \text { for all } 0<t \leqslant 1,
$$

then, for any $\lambda_{0}>0$, if $\lambda>\lambda_{0}$ and $\lambda-\lambda_{0}$ is small enough

$$
\begin{aligned}
& \frac{\sqrt{\lambda_{0}}}{\sqrt{\lambda}} u_{\lambda} \leqslant u_{\lambda_{0}}, \\
& \text { int } N_{\lambda} \supset N_{\lambda_{0}} .
\end{aligned}
$$

Proof. Set $\theta=\left(\lambda_{0} / \lambda\right)^{1 / 2}$. Then $\Delta\left(\theta u_{\lambda}\right)=h\left(u_{\lambda}\right) \lambda_{0} f\left(\theta u_{\lambda}\right)$ where

$$
h(t)=\frac{\theta \lambda}{\lambda_{0}} \frac{f(t)}{f(\theta t)}=\frac{1}{\theta} \frac{f(t)}{f(\theta t)}>1
$$

by (7.31), provided $\theta<1,1-\theta$ is small enough. Thus

$$
\Delta\left(\theta u_{\lambda}\right)>\lambda_{0} f\left(\theta u_{\lambda}\right), \quad \Delta u_{\lambda_{0}}=\lambda_{0} f\left(u_{\lambda_{0}}\right) .
$$

By Corollary 1.2 (working with $\left(1-u_{\lambda_{0}}\right)^{1 / \beta},\left(1-\theta u_{\lambda}\right)^{1 / \beta}$ ) we find that $\theta u_{\lambda}-u_{\lambda_{0}}$ cannot take positive maximum in $\Omega$. Thus, if (7.32) is not true then there is a point 
$X^{0} \in \partial \Omega$ where $\theta u_{\lambda}-u_{\lambda_{0}}$ attains its positive maximum in $\bar{\Omega}$, and

$$
\theta u_{\lambda}>u_{\lambda_{0}}, \quad \theta \frac{\partial u_{\lambda}}{\partial \nu} \geqslant \frac{\partial u_{\lambda_{0}}}{\partial \nu} \text { at } X^{0}
$$

But then, at $X^{0}$,

$$
\begin{aligned}
0 & =\frac{1}{\sqrt{\lambda_{0}}} \frac{\partial u_{\lambda_{0}}}{\partial \nu}+\mu\left(u_{\lambda_{0}}-1\right)<\frac{\theta}{\sqrt{\lambda_{0}}} \frac{\partial u_{\lambda}}{\partial \nu}+\mu\left(\theta u_{\lambda}-1\right) \\
& <\frac{1}{\sqrt{\lambda}} \frac{\partial u_{\lambda}}{\partial \nu}+\mu\left(u_{\lambda}-1\right)=0, \text { a contradiction. }
\end{aligned}
$$

Having proved (7.32), the proof of (7.33) is then similar to the proof of Theorem 1.6 .

We can now state the main result of this section.

THEOREM 7.8. Assume that (7.6) and (2.1), (7.31) hold. Then there exists a $\lambda_{*}>0$ such that $N_{\lambda}$ is a closed convex domain with $C^{1+\beta}$ boundary for any $\lambda>\lambda_{*}, N_{\lambda_{*}}$ consists of a single point, and $N_{\lambda}=\varnothing$ if $\lambda<\lambda_{*}$.

Proof. For $\lambda$ large enough Lemma 7.5 implies the flatness condition and thus, by [1], $\partial N_{\lambda}$ is a $C^{1+\beta}$ curve which can be parametrized by $\partial \Omega$. Lemma 7.4 then shows that $N_{\lambda}$ is a convex domain. We can now use the monotonicity of $N_{\lambda}$ (in $\lambda$ ) established in Lemma 7.7 in order to proceed as in the proof of Theorem 5.3. Notice finally that $N_{0}=\varnothing$, by the maximum principle; hence $N_{\lambda}=\varnothing$ if $\lambda$ is small enough, that is, $\lambda_{*}>0$.

\section{REFERENCES}

1. H. W. Alt and D. Phillips, A free boundary problem for semilinear elliptic equations (to appear).

2. R. Aris, The mathematical theory of diffusion and reaction in permeable catalysts, Clarendon Press, Oxford, 1975.

3. C. Bandle, R. P. Sperb and I. Stakgold, The single steady state irreverisble reaction (to appear).

4. L. A. Caffarelli and I. Spruck, Convexity properties of solutions of some classical variational problems, Comm. Partial Differential Equations 7 (1982), 1337-1379.

5. D. S. Cohen and T. W. Laetsch, Nonlinear boundary value problems suggested by Chemical reactor theory, J. Differential Equations 7 (1970), 217-226.

6. R. Courant and D. Hilbert, Methods of mathematical physics, II: Partial differential equations, Interscience, New York, 1962.

7. J. I. Diaz and J. Hernandez, On the existence of a free boundary for a class of reaction-diffusion systems (to appear).

8. H. Federer, Geometric measure theory, Springer-Verlag, Berlin, 1969.

9. E. Giusti, Minimal surfaces and functions of bounded variations, Notes on Pure Mathematics, Australian National University, Canberra, 1977.

10. H. B. Keller, Elliptic boundary value problems suggested by nonlinear diffusion processes, Arch. Rational Mech. Anal. 35 (1969), 363-381.

11. H. Lewy and G. Stampacchia, On the regularity of the solution of a variational inequality, Comm. Pure Appl. Math. 22 (1969), 153-188.

12. J. Mossino, A priori estimates for a model of Grad-Mercier type in plasma confinement, Applicable Anal. 13 (1982), 185-207.

13. L. E. Payne and G. A. Philippin, Some maximum principles for nonlinear elliptic equations in divergence form with applications to capillary surfaces and to surfaces of constant mean curvature, Nonlinear Anal. 3 (1979). 
14. L. E. Payne and I. Stakgold, On the mean value of the fundamental mode in the fixed membrane problem, Applicable Anal. 3 (1973), 295-303.

15. D. Phillips, A minimization problem and the regularity of solutions in the presence of a free boundary, Indiana Univ. Math. J. 32 (1983), 1-17.

16. Hausdorff measure estimates of a free boundary for a minimum problem, Comm. Partial Differential Equations 8 (1983), 1409-1454.

17. R. Sperb and I. Stakgold, Estimates for membranes of varying density, Applicable Anal. 8 (1979), 301-318.

18. I. Stakgold, Gradient bounds for plasma confinement, Math. Methods Appl. Sci. 2 (1980), 68-72.

19. __ Estimates for some free bounary problems, Ordinary and Partial Differential Equations, Lecture Notes in Math., vol. 846, Springer-Verlag, Berlin and New York, 1982.

Department of Mathematics, NorthWestern University, Evanston, IllinOis 60201

Department of Mathematics, Purdue University, Lafayette, Indiana 47907 